\title{
Competition of three species in an advective environment
}

\author{
Olga Vasilyeva* $\quad$ Frithjof Lutscher ${ }^{\dagger \ddagger}$
}

December 22, 2011

\begin{abstract}
Individuals in advective environments, for example rivers, coastlines, or the gut, are subject to movement with directional bias We study how this movement bias shapes community composition by considering how the strength of movement bias affects the outcome of competition among three species. Our model is a system of three reaction-advection-diffusion equations with Danckwerts' boundary conditions. Our key tool in this study is to use the dominant eigenvalue of the diffusionadvection operator in order to reduce the spatially explicit model to a spatially implicit ordinary differential equation model. After an in-depth analysis of the implicit model, we use numerical simulations of the explicit model to test the predictions obtained from the analysis. In general, we find a good qualitative agreement between the explicit and the implicit model. We find that varying the strength of advection can fundamentally alter the outcome of competition between the three species, and we characterize the possible transitions. In particular, water extraction and flow control can destabilize existing species communities or facilitate invasions of non-native species.
\end{abstract}

AMS classification: $35 \mathrm{~K} 57,92 \mathrm{D} 40,37 \mathrm{C} 75$

Keywords: river ecosystem, competition, coexistence, reaction-advection-diffusion,

\footnotetext{
${ }^{*}$ Department of Mathematics and Statistics, University of Ottawa, Ottawa, ON K1N8G1

${ }^{\dagger}$ Department of Mathematics and Statistics, University of Ottawa, 585 King Edward Avenue, Ottawa, ON K1N8G1, supported by an NSERC Discovery grant and an Early Researcher Award from the MRI, Ontario.

${ }^{\ddagger}$ Corresponding author. email: flutsche@uottawa.ca. phone: ++1 613-562-5800 ext. 3510
} 


\section{Introduction}

Competition is ubiquitous among species; it is an essential mechanism in determining species richness and shaping communities. Simple models for resource competition predict competitive exclusion of all but the most efficient competitor, while Lotka-Volterra competition models allow for coexistence if interspecific competition is weaker than intraspecific competition, see e.g. [16]. Considering the abundance of coexisting species in most parts of the world, ecologists have searched for, and found, a number of mechanisms that lead to coexistence, many of which involve some form of spatial trade-offs, e.g. spatial niches and segregation, competition-colonization trade-offs, spatio-temporal heterogeneity, or metapopulation dynamics, see e.g. [6, 17, 10]. A fairly recent theoretical observation is that advection can also facilitate coexistence of competitors [1, 19, 24].

Advection is the bias of individual movement in a given direction. Most obviously, it occurs in rivers where individuals are transported downstream by the water flow. The idea that random unbiased movement could prevent wash-out of such populations is the basis of a number of recent studies [27, 20, 22]. Other advective environments are coastlines with unidirectional currents [25], the gut $[1,5]$, the water column in a lake [14], mountain slopes [26], and, after a suitable change of coordinates, even moving temperature isoclines $[4,24]$. In this work, we will typically refer to rivers, but the theory applies to all cases.

Whether one considers resource competition or Lotka-Volterra competition, a common result that emerges from previous work is that advection can change the outcome of competition between two species and allow for stable coexistence $[1,12,19,24,29]$. We use and build on these results to study the effect of advection on three competing species. We are particularly interested in the question of when and how advection allows for coexistence of competitors that would not coexist without advection, and, vice versa, when and how advection can destroy a coexistence scenario and lead to species extinction. This question is particularly interesting as flow rates and discharge in more and more rivers are regulated and controlled by human needs. Increasing water and power demands typically lead to changes in flow rates. We need to understand how these changes in flow rates can affect the associated river ecosystem in order to maintain its function and integrity.

The dynamics of a non-spatial Lotka-Volterra competition model for three species are much richer than for the two-species counterpart, as the three-dimensional system is generally not monotone [13]. For example, Zeeman classified the behavior of three-dimensional Lotka-Volterra models by isolating 33 possible cases, eight of which involve limit cycles [32], more results are available in [11, 21, 30, 31, 33]. There are only a few results on spatial three-species competition models in the absence of advection, namely, conditions for permanence of the system [9, 8], and diffusion-driven instabilities [15]. The only models to include advection that we are aware of are by Ballyk and coworkers (e.g. [1]). While these 
are formulated for an arbitrary number of species, we are not aware of any coexistence results for more than two species.

We formulate our model in the next section. We reduce the number of parameters through a few simplifying assumptions that focus on the question of how advection affects coexistence of three (rather than two) species. Specifically, we concentrate on two cases, called Cyclic and Transitive (see Section 2). Obviously, the analysis of a three-dimensional reaction-diffusion system is quite difficult. As a first step, we reduce the spatially explicit reaction-advection diffusion model to a spatially implicit ordinary differential equation model. In Section 3, we collect some known results on three-species competition system that we use throughout our analysis. We analyze our two models in detail (Sections 4 and 5), and we use the results as a guide for numerical explorations of the spatially explicit model in Section 6 . In the final discussion, we relate our results back to the biological application, and we suggest future research areas.

\section{Model}

We consider the following reaction-diffusion-advection model for three competing species

$$
\left\{\begin{array}{l}
\frac{\partial u_{1}}{\partial t}=d \frac{\partial^{2} u_{1}}{\partial x^{2}}-q \frac{\partial u_{1}}{\partial x}+u_{1}\left(r_{1}-a_{11} u_{1}-a_{12} u_{2}-a_{13} u_{3}\right), \\
\frac{\partial u_{2}}{\partial t}=d \frac{\partial^{2} u_{2}}{\partial x^{2}}-q \frac{\partial u_{2}}{\partial x}+u_{2}\left(r_{2}-a_{21} u_{1}-a_{22} u_{2}-a_{23} u_{3}\right) \\
\frac{\partial u_{3}}{\partial t}=d \frac{\partial^{2} u_{3}}{\partial x^{2}}-q \frac{\partial u_{3}}{\partial x}+u_{3}\left(r_{3}-a_{31} u_{1}-a_{32} u_{2}-a_{33} u_{3}\right)
\end{array}\right.
$$

where $u_{i}(t, x)$ is the density of the $i$-th species at time $t$ at point $x$ of the bounded domain $[0, L]$. For simplicity, we assume that the diffusion coefficient, $d>0$, and the effective advection speed, $q \geq 0$, are the same for all three species. We return to this point in the discussion. The intrinsic growth rates, $r_{i}$, and the inter- and intra-specific competition coefficients, $a_{i j}$ are assumed positive. This model is the natural generalization of the two-competitor model considered in [19].

We impose so-called Danckwerts' boundary conditions [2]

$$
d \frac{\partial u_{i}}{\partial x}(t, 0)-q u_{i}(t, 0)=0, \quad \frac{\partial u_{i}}{\partial x}(t, L)=0 .
$$

The reflecting boundary condition at $x=0$ indicates that individuals cannot cross the upstream boundary and move beyond the top of the stream. The downstream condition indicates that net out-flux from the domain is due to advection only and not to diffusion.

It was shown numerically in [19] and partially confirmed analytically in [29], that an increase in advection may change the competitive outcome in a two-species Lotka-Volterra spatial model. Namely, the weaker competitor (in the case of low advection) wins in the case of faster flow, provided it has a higher intrinsic growth rate. Thus, to make our model a natural extension of the two-species setting, 
we assume that in the absence of advection $(q=0)$, in each of the two species subsystems, one species competitively excludes the other. This restriction leaves only two possible arrangements between three competitors when $q=0$ (up to permutation): (i) the cyclic case, known from the "rock-paper-scissors" game; and (ii) the transitive case where one species wins against both competitors.

The intrinsic growth rates, $r_{i}$, of the competitors are assumed to be different. Then there are two subcases of the cyclic arrangement: "clockwise" and "counterclockwise", which we treat in Section 4. The transitive case is more complex as it allows six possible arrangements of different $r_{i}$. Since advection can change the outcome of two-species competition only if the weaker competitor has the higher intrinsic growth rate $[19,29]$, we focus only the case where the weakest competitor has the highest growth rate and the strongest competitor has the lowest growth rate, see Section 5 .

When analyzing a model as complex as system (2.1), one has a choice between exact results that cannot be expressed in a simple and closed form and simple explicit expressions that are only approximations. For example, one can give exact stability and invasion conditions of various one- and two-species equilibria in terms of variational formulae [7], but these can only be evaluated numerically. We choose the second approach and replace the diffusion-advection term in each of the three equations by $\lambda u_{i}$, where $\lambda$ is the leading eigenvalue of the diffusion-advection operator subject to our boundary conditions. With this replacement, the reaction-diffusion system turns into a spatially implicit system of ordinary differential equations

$$
\left\{\begin{array}{l}
\frac{d u_{1}}{d t}=\lambda u_{1}+u_{1}\left(r_{1}-a_{11} u_{1}-a_{12} u_{2}-a_{13} u_{3}\right), \\
\frac{d u_{2}}{d t}=\lambda u_{2}+u_{2}\left(r_{2}-a_{21} u_{1}-a_{22} u_{2}-a_{23} u_{3}\right), \\
\frac{d u_{3}}{d t}=\lambda u_{3}+u_{3}\left(r_{3}-a_{31} u_{1}-a_{32} u_{2}-a_{33} u_{3}\right)
\end{array}\right.
$$

where $u_{i}=u_{i}(t)$ now depends only on the time variable. Since $1 /|\lambda|$ denotes the average time that an individual spends in the domain before being washed out [1], this replacement can be viewed in some sense as spatial averaging of the movement part of the equation, the linear differential operator. The nonlinear reaction part of the equation cannot be averaged easily. Nonetheless, this simplification turns out to be a very useful tool in the analysis of the behavior of the spatial model.

Various comparisons and numerical results show that the spatially implicit model gives a very close description of the behavior of the spatially explicit model in the case of a single or two competing species [29], and also in predator-prey systems [28]. A similar technique known as average dispersal success approximation, has been shown to work equally well in discrete-time equations, and can be derived more formally in terms of Taylor series [18]. To our knowledge, no formal derivations exist in the case of reaction-diffusion equations.

Standard techniques, such as separation of variables, show that the leading eigenvalue $\lambda=\lambda(d, q)$ of the equation $d u^{\prime \prime}-q u^{\prime}=\lambda u$ with boundary conditions (2.2) is a non-positive, decreasing function 
of $q$ for fixed $d$, and $\lambda(d, 0)=0$. Thus, in order to study the behavior of the spatial model (2.1) as $q$ increases from zero to its maximum value $q^{*}=2 \sqrt{d \max \left(r_{i}\right)}$, we study the behavior of (2.3) as $\lambda$ decreases from $\lambda=0$ to its minimal value $\lambda=-\max \left(r_{i}\right)$.

\section{Preliminaries}

In our analysis of model (2.3), we frequently use two important theorems about three-species competition models. To make this work self contained, we cite these results here. We begin with some definitions.

A two-species Lotka-Volterra competition system

$$
\frac{d y_{1}}{d t}=y_{1}\left(r_{1}-a_{11} y_{1}-a_{12} y_{2}\right), \quad \frac{d y_{2}}{d t}=y_{2}\left(r_{2}-a_{21} y_{1}-a_{22} y_{2}\right)
$$

exhibits one of four qualitatively different behaviors, depending on parameters, namely

- stable coexistence, if $r_{1}>\frac{a_{12} r_{2}}{a_{22}}$ and $r_{2}>\frac{a_{21} r_{1}}{a_{11}}$

- competitive exclusion by first species, if $r_{1}>\frac{a_{12} r_{2}}{a_{22}}$ and $r_{2}<\frac{a_{21} r_{1}}{a_{11}}$;

- competitive exclusion by second species, if $r_{1}<\frac{a_{12} r_{2}}{a_{22}}$ and $r_{2}>\frac{a_{21} r_{1}}{a_{11}}$;

- bistability or founder control, if $r_{1}<\frac{a_{12} r_{2}}{a_{22}}$ and $r_{2}<\frac{a_{21} r_{1}}{a_{11}}$.

The general three-species competition model reads

$$
\left\{\begin{array}{c}
\frac{d y_{1}}{d t}=y_{1}\left(r_{1}-a_{11} y_{1}-a_{12} y_{2}-a_{13} y_{3}\right), \\
\frac{d y_{2}}{d t}=y_{2}\left(r_{2}-a_{21} y_{1}-a_{22} y_{2}-a_{23} y_{3}\right), \\
\frac{d y_{3}}{d t}=y_{3}\left(r_{3}-a_{31} y_{1}-a_{32} y_{2}-a_{33} y_{3}\right),
\end{array}\right.
$$

where $r_{i}>0$ and $a_{i j}>0$ have the same meaning as above. We denote the matrix of competition coefficients as $A=\left(a_{i j}\right)$.

This system has at least four fixed points: one unpopulated fixed point $(0,0,0)$ and three singlespecies fixed points $F_{1}=\left(\frac{r_{1}}{a_{11}}, 0,0\right), F_{2}=\left(0, \frac{r_{2}}{a_{22}}, 0\right), F_{3}=\left(0,0, \frac{r_{3}}{a_{33}}\right)$. Since all parameters are assumed positive, solutions of (3.2) are uniformly bounded, see e.g. Theorem 15.2.4 of [13].

System (3.2) is called persistent, if solutions with positive initial conditions in all coordinates satisfy $\lim \sup y_{i}(t)>0$. System $(3.2)$ is called permanent, if solutions with positive initial conditions in all coordinates are uniformly bounded from below, i.e. $\lim \inf y_{i}(t)>\delta$ for some $\delta>0$. System (3.2) admits a heteroclinic cycle, if each of its two-species subsystems has a competitive exclusion outcome, and there is a cyclic arrangement of "winners" and "losers", i.e. in the absence of species $i$ species $i-1$ will outcompete species $i-2$ (indices are counted modulo 3 ). Thus, there are orbits in the 
three coordinate planes in $y_{1} y_{2} y_{3}$-space, with the $i$ th orbit having $\alpha$-limit $F_{i}$ and $\omega$-limit $F_{i+1}$ (indices modulo 3). A heteroclinic cycle occurs if

$$
\frac{r_{1}}{r_{2}}<\frac{a_{12}}{a_{22}}, \frac{a_{11}}{a_{21}} ; \quad \frac{r_{2}}{r_{3}}<\frac{a_{23}}{a_{33}}, \frac{a_{22}}{a_{32}} ; \quad \frac{r_{3}}{r_{1}}<\frac{a_{31}}{a_{11}}, \frac{a_{33}}{a_{13}} .
$$

We now state the two theorems that we frequently use throughout this paper.

Theorem 3.1 ([13], p. 206). System (3.2) is persistent and uniformly bounded iff

1. it has an interior fixed point $\bar{y}^{*}$;

2. $\operatorname{det}(A)>0$;

3. none of the two-species subsystems of (3.2) has the founder control outcome (see Remark 3.2).

Remark 3.2. Note that system (3.1) has the founder control outcome iff

$$
\frac{a_{11}}{a_{21}}<\frac{r_{1}}{r_{2}}<\frac{a_{12}}{a_{22}} .
$$

This inequality implies $a_{11} a_{22}-a_{12} a_{21}<0$. Thus, if $\operatorname{det}\left(\begin{array}{ll}a_{11} & a_{12} \\ a_{21} & a_{22}\end{array}\right)>0$, then founder control is not possible, no matter what the growth rates are.

Theorem 3.3 ([13], p. 207). System (3.2) is permanent iff it satisfies conditions (1-3) above, and in the case where it admits a heteroclinic cycle, the coefficients satisfy

$$
\left(\frac{a_{31} r_{1}}{a_{11} r_{3}}-1\right)\left(\frac{a_{12} r_{2}}{a_{22} r_{1}}-1\right)\left(\frac{a_{23} r_{3}}{a_{33} r_{2}}-1\right)<\left(1-\frac{a_{21} r_{1}}{a_{11} r_{2}}\right)\left(1-\frac{a_{32} r_{2}}{a_{22} r_{3}}\right)\left(1-\frac{a_{13} r_{3}}{a_{33} r_{1}}\right),
$$

or, equivalently,

$$
\begin{array}{r}
\left(a_{31} r_{1}-a_{11} r_{3}\right)\left(a_{12} r_{2}-a_{22} r_{1}\right)\left(a_{23} r_{3}-a_{33} r_{2}\right) \\
<\quad\left(a_{11} r_{2}-a_{21} r_{1}\right)\left(a_{22} r_{3}-a_{32} r_{2}\right)\left(a_{33} r_{1}-a_{13} r_{3}\right) .
\end{array}
$$

If the system is permanent, any solution will either approach the interior fixed point (in case it is stable), or a limit cycle (if the interior fixed point is unstable).

\section{Cyclic case}

We begin our analysis of model (2.3) with the so-called cyclic case; i.e. when the system admits a heteroclinic cycle for $\lambda=0$. If we assume that the growth rates are ordered as $r_{1}>r_{2}>r_{3}>0$, then we have two sub-cases of the cyclic case. In case I, species $i$ outcompetes species $i-1$ (modulo 3 ), whereas in case II, species $i$ outcompetes species $i+1$ (modulo 3 ). 
In order to reduce the number of parameters in our model, we assume that the competition matrix in (3.2) has the form

$$
A=\left(\begin{array}{ccc}
r_{1} & r_{1} \alpha & r_{1} \beta \\
r_{2} \beta & r_{2} & r_{2} \alpha \\
r_{3} \alpha & r_{3} \beta & r_{3}
\end{array}\right),
$$

where $0<\beta<1<\alpha$ (case I) or $0<\alpha<1<\beta$ (case II), and $\alpha \beta<1$. The first condition (in both cases) ensures the "rock-paper-scissors" arrangement, and the second assumption excludes the possibility of founder control in any of the two-species subsystems by Remark 3.2.

Now, System (2.3) can be rewritten as

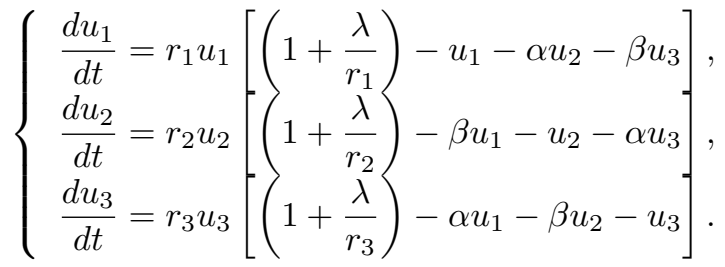

Our first goal is to investigate persistence of system (4.2) as we vary $\lambda \leq 0$. Thus, we need to check the three conditions in Theorem 3.1. We start with the following observation.

Lemma 4.1. (a) $\alpha^{2}-\alpha \beta+\beta^{2}-\alpha-\beta+1>0$.

$$
\text { (b) } \Delta:=\operatorname{det}\left(\begin{array}{ccc}
1 & \alpha & \beta \\
\beta & 1 & \alpha \\
\alpha & \beta & 1
\end{array}\right)>0 \text {. }
$$

Proof. (a) We have $\alpha^{2}-\alpha \beta+\beta^{2}-\alpha-\beta+1=\frac{3}{4}(\alpha-\beta)^{2}+\frac{1}{4}(\alpha+\beta-2)^{2}>0$.

(b) The determinant is given by

$$
\begin{aligned}
\alpha^{3}+\beta^{3}-3 \alpha \beta+1 & =(\alpha+\beta)^{3}-3 \alpha^{2} \beta-3 \alpha \beta^{2}-3 \alpha \beta+1 \\
& =(\alpha+\beta)^{3}+1-3 \alpha \beta(\alpha+\beta+1) \\
& =(\alpha+\beta+1)\left(\alpha^{2}+2 \alpha \beta+\beta^{2}-\alpha-\beta+1\right)-3 \alpha \beta(\alpha+\beta+1) \\
& =(\alpha+\beta+1)\left(\alpha^{2}-\alpha \beta+\beta^{2}-\alpha-\beta+1\right) .
\end{aligned}
$$

The statement now follows from $\alpha+\beta+1>0$ and (a).

In a series of lemmas, we determine conditions for which model (4.2) has an interior fixed point, i.e. a fixed point with all coordinates positive.

Lemma 4.2. (a) For $\lambda=0$, system (4.2) admits a unique interior fixed point $\left(u_{1}^{0}, u_{2}^{0}, u_{3}^{0}\right)=(\gamma, \gamma, \gamma)$, where $\gamma=\frac{1}{1+\alpha+\beta}$. 
(b) For $\lambda<0$, system (4.2) admits a unique fixed point $\left(u_{1}^{\lambda}, u_{2}^{\lambda}, u_{3}^{\lambda}\right)$ with

$$
\begin{aligned}
& u_{1}^{\lambda}=\gamma+\frac{\lambda}{\Delta}\left(\frac{1-\alpha \beta}{r_{1}}+\frac{\beta^{2}-\alpha}{r_{2}}+\frac{\alpha^{2}-\beta}{r_{3}}\right), \\
& u_{2}^{\lambda}=\gamma+\frac{\lambda}{\Delta}\left(\frac{1-\alpha \beta}{r_{2}}+\frac{\beta^{2}-\alpha}{r_{3}}+\frac{\alpha^{2}-\beta}{r_{1}}\right), \\
& u_{3}^{\lambda}=\gamma+\frac{\lambda}{\Delta}\left(\frac{1-\alpha \beta}{r_{3}}+\frac{\beta^{2}-\alpha}{r_{1}}+\frac{\alpha^{2}-\beta}{r_{2}}\right),
\end{aligned}
$$

and $\Delta$ as in Lemma 4.1.

Proof. (a) Clearly, $\left(u_{1}^{0}, u_{2}^{0}, u_{3}^{0}\right)$ is an interior fixed point. Uniqueness follows from Lemma 4.1(b).

(b) Follows from Cramer's rule and Lemma 4.1(b).

Proposition 4.3. System (4.2) is persistent iff all three of the following inequalities hold:

$$
\begin{aligned}
& \lambda\left(\frac{1-\alpha \beta}{r_{1}}+\frac{\beta^{2}-\alpha}{r_{2}}+\frac{\alpha^{2}-\beta}{r_{3}}\right)+\alpha^{2}-\alpha \beta+\beta^{2}-\alpha-\beta+1>0, \\
& \lambda\left(\frac{1-\alpha \beta}{r_{2}}+\frac{\beta^{2}-\alpha}{r_{3}}+\frac{\alpha^{2}-\beta}{r_{1}}\right)+\alpha^{2}-\alpha \beta+\beta^{2}-\alpha-\beta+1>0, \\
& \lambda\left(\frac{1-\alpha \beta}{r_{3}}+\frac{\beta^{2}-\alpha}{r_{1}}+\frac{\alpha^{2}-\beta}{r_{2}}\right)+\alpha^{2}-\alpha \beta+\beta^{2}-\alpha-\beta+1>0 .
\end{aligned}
$$

Proof. Lemma 4.1 implies that the second condition in Theorem 3.1 is satisfied for any $\lambda$. As we noted above, the condition $\alpha \beta<1$ excludes the founder control outcome in any of the three two-species subsystems, as long as $\lambda>-r_{3}$ (see Remark 3.2), hence the third condition in Theorem 3.1 is true as well. Thus, the only condition we have to check is that the fixed point is indeed in $\mathbb{R}_{+}^{3}$. The rest follows by Lemma $4.2(\mathrm{~b})$.

Note that if System (4.2) is persistent, then it has an interior fixed point $\left(u_{1}^{\lambda}, u_{2}^{\lambda}, u_{3}^{\lambda}\right)$, with $u_{i}^{\lambda}>0$ for $i=1,2,3$. Thus, by definition of the interior fixed point, we have from (4.2)

$$
\left(\begin{array}{lll}
1 & \alpha & \beta \\
\beta & 1 & \alpha \\
\alpha & \beta & 1
\end{array}\right)\left(\begin{array}{l}
u_{1}^{\lambda} \\
u_{2}^{\lambda} \\
u_{3}^{\lambda}
\end{array}\right)=\left(\begin{array}{c}
1+\frac{\lambda}{r_{1}} \\
1+\frac{\lambda}{r_{2}} \\
1+\frac{\lambda}{r_{3}}
\end{array}\right) .
$$

Since all components of the matrix are positive, the right-hand side above has positive components as well. Thus, in order for system to be persistent, the value of $\lambda$ has to be greater than $-r_{3}$.

By Lemma 4.1(a), if $\lambda=0$ then inequalities (4.3), (4.4), (4.5) hold, and we have an interior fixed point. In addition, each of the inequalities is linear with respect to $\lambda$. Therefore, there exists a persistence boundary $\lambda_{c}<0$ such that at least one of the inequalities becomes an equality when $\lambda=\lambda_{c}$, but all three inequalities hold (and the system is persistent) for $\lambda \in\left(\lambda_{c}, 0\right]$. Necessarily, we have $\lambda_{c}>-r_{3}$.

In the following two subsections, we find explicit formulas for $\lambda_{c}$ in the two cases. 


\subsection{Persistence in case I}

Here we assume $0<\beta<1<\alpha$. We start by proving the following technical lemmas.

Lemma 4.4. Assume that $0<\beta<1<\alpha, \alpha \beta<1$ and $r_{1}>r_{2}>r_{3}>0$. Then the following inequalities hold

(a) $\frac{1-\alpha \beta}{r_{2}}+\frac{\beta^{2}-\alpha}{r_{3}}+\frac{\alpha^{2}-\beta}{r_{1}}<\frac{\alpha^{2}-\alpha \beta+\beta^{2}-\alpha-\beta+1}{r_{3}}$;

(b) $\frac{1-\alpha \beta}{r_{3}}+\frac{\beta^{2}-\alpha}{r_{1}}+\frac{\alpha^{2}-\beta}{r_{2}}>\frac{\alpha^{2}-\alpha \beta+\beta^{2}-\alpha-\beta+1}{r_{1}}>0$.

Proof. The result follows from $\alpha>1>\beta>0$ and $r_{1}>r_{2}>r_{3}$, and Lemma 4.1(a).

Lemma 4.5. Inequality (4.4) holds for $\lambda=\lambda_{c}$, i.e.

$$
\lambda_{c}\left(\frac{1-\alpha \beta}{r_{2}}+\frac{\beta^{2}-\alpha}{r_{3}}+\frac{\alpha^{2}-\beta}{r_{1}}\right)+\alpha^{2}-\alpha \beta+\beta^{2}-\alpha-\beta+1>0 .
$$

Proof. Since $-r_{3}<\lambda_{c}<0$, by Lemma 4.4(a) and Lemma 4.1(a) we get

$$
\begin{aligned}
& \lambda_{c}\left(\frac{1-\alpha \beta}{r_{2}}+\frac{\beta^{2}-\alpha}{r_{3}}+\frac{\alpha^{2}-\beta}{r_{1}}\right)+\alpha^{2}-\alpha \beta+\beta^{2}-\alpha-\beta+1 \\
>\quad & \lambda_{c} \frac{\alpha^{2}-\alpha \beta+\beta^{2}-\alpha-\beta+1}{r_{3}}+\alpha^{2}-\alpha \beta+\beta^{2}-\alpha-\beta+1 \\
>\quad & -r_{3} \frac{\alpha^{2}-\alpha \beta+\beta^{2}-\alpha-\beta+1}{r_{3}}+\alpha^{2}-\alpha \beta+\beta^{2}-\alpha-\beta+1=0 .
\end{aligned}
$$

Proposition 4.6. The critical value for persistence, $\lambda_{c}$, in Case I is given by

$$
\lambda_{c}=-\frac{\alpha^{2}-\alpha \beta+\beta^{2}-\alpha-\beta+1}{\max \left(\lambda_{c 1}, \lambda_{c 2}\right)},
$$

where

$$
\lambda_{c 1}=\frac{1-\alpha \beta}{r_{1}}+\frac{\beta^{2}-\alpha}{r_{2}}+\frac{\alpha^{2}-\beta}{r_{3}}
$$

and

$$
\lambda_{c 2}=\frac{1-\alpha \beta}{r_{3}}+\frac{\beta^{2}-\alpha}{r_{1}}+\frac{\alpha^{2}-\beta}{r_{2}} .
$$

Proof. By definition of $\lambda_{c}$, one of inequalities (4.3), (4.4) and (4.5) must turn into an equality. Lemma 4.5 excludes it for (4.4). The formula now follows from the fact that for $\lambda=\lambda_{c}$, the left side of either (4.3) or (4.5) turns into zero. 


\subsection{Persistence in case II}

Now we assume $0<\beta<1<\alpha$ and get the critical threshold for persistence, $\lambda_{c}$.

Lemma 4.7. Assume that $0<\alpha<1<\beta, \alpha \beta<1$ and $r_{1}>r_{2}>r_{3}>0$. Then the following inequalities hold

(a) $\frac{1-\alpha \beta}{r_{2}}+\frac{\beta^{2}-\alpha}{r_{3}}+\frac{\alpha^{2}-\beta}{r_{1}}>0$;

(b) $\frac{1-\alpha \beta}{r_{1}}+\frac{\beta^{2}-\alpha}{r_{2}}+\frac{\alpha^{2}-\beta}{r_{3}}<\frac{1-\alpha \beta}{r_{2}}+\frac{\beta^{2}-\alpha}{r_{3}}+\frac{\alpha^{2}-\beta}{r_{1}}$;

(c) $\frac{1-\alpha \beta}{r_{3}}+\frac{\beta^{2}-\alpha}{r_{1}}+\frac{\alpha^{2}-\beta}{r_{2}}<\frac{1-\alpha \beta}{r_{2}}+\frac{\beta^{2}-\alpha}{r_{3}}+\frac{\alpha^{2}-\beta}{r_{1}}$.

Proof. (a) It follows from the assumptions that

$$
\frac{1-\alpha \beta}{r_{2}}+\frac{\beta^{2}-\alpha}{r_{3}}+\frac{\alpha^{2}-\beta}{r_{1}}>\frac{1-\alpha \beta}{r_{1}}+\frac{\beta^{2}-\alpha}{r_{1}}+\frac{\alpha^{2}-\beta}{r_{1}}=\frac{\alpha^{2}-\alpha \beta+\beta^{2}-\alpha-\beta+1}{r_{1}}>0 .
$$

(b) The inequality holds term-by-term.

(c) Taking the difference, we get

$$
\begin{aligned}
& \frac{1-\alpha \beta}{r_{2}}+\frac{\beta^{2}-\alpha}{r_{3}}+\frac{\alpha^{2}-\beta}{r_{1}}-\frac{1-\alpha \beta}{r_{3}}-\frac{\beta^{2}-\alpha}{r_{1}}-\frac{\alpha^{2}-\beta}{r_{2}} \\
= & \frac{\alpha^{2}-\beta-\beta^{2}+\alpha}{r_{1}}+\frac{1-\alpha \beta-\alpha^{2}+\beta}{r_{2}}+\frac{\beta^{2}-\alpha-1+\alpha \beta}{r_{3}} \\
= & \frac{(\alpha-\beta)(\alpha+\beta+1)}{r_{1}}+\frac{(1-\alpha)(\alpha+\beta+1)}{r_{2}}+\frac{(\beta-1)(\alpha+\beta+1)}{r_{3}} \\
> & \frac{(\alpha-\beta)(\alpha+\beta+1)+(1-\alpha)(\alpha+\beta+1)+(\beta-1)(\alpha+\beta+1)}{r_{1}}=0,
\end{aligned}
$$

as needed.

Lemma 4.7 implies that, in Case II, inequality (4.4) is always the first to fail when we decrease $\lambda<0$. Thus, we get

Proposition 4.8. The critical value of $\lambda$ for persistence in Case II is given by

$$
\lambda_{c}=-\frac{\alpha^{2}-\alpha \beta+\beta^{2}-\alpha-\beta+1}{\frac{1-\alpha \beta}{r_{2}}+\frac{\beta^{2}-\alpha}{r_{3}}+\frac{\alpha^{2}-\beta}{r_{1}}} .
$$

\subsection{Permanence and Stability}

We now turn our attention to the question of permanence. By Theorem 3.3, with our assumptions, System (4.2) with $\lambda=0$ is permanent iff it is persistent and satisfies $(\alpha-1)^{3}<(1-\beta)^{3}$, or, equivalently, $\alpha+\beta<2$. Note that if the system is permanent, then either the interior fixed point is stable, or it is unstable and there exists a stable interior limit cycle. 
We investigate the stability of the interior fixed point when $\lambda=0$ and when $\lambda$ approaches $\lambda_{c}$ (i.e. the interior fixed point approaches the boundary). First, we show that if $\lambda=0$ and the permanence condition $\alpha+\beta<2$ is satisfied, then the interior fixed point is stable (so there is no stable limit cycle). Namely, we prove the following.

Proposition 4.9. Suppose $r_{1}, r_{2}, r_{3}>0, \alpha, \beta>0$ and $\alpha+\beta<2$. Then the interior fixed point $(\gamma, \gamma, \gamma)$ of system (4.2) with $\lambda=0$ is asymptotically stable.

Proof. The Jacobian at the fixed point is given by

$$
J_{r_{1} r_{2} r_{3}}=\frac{1}{1+\alpha+\beta}\left(\begin{array}{ccc}
-r_{1} & -r_{1} \alpha & -r_{1} \beta \\
-r_{2} \beta & -r_{2} & -r_{2} \alpha \\
-r_{3} \alpha & -r_{3} \beta & -r_{3}
\end{array}\right) .
$$

In the case $r_{1}=r_{2}=r_{3}=r>0$, the eigenvalues of $J$ have negative real parts. Namely, $J_{r r r}$ has eigenvalue $\mu_{1}=-r$ (with eigenvector $(1,1,1)$ ) and trace $\operatorname{tr}(J)=-\frac{3 r}{1+\alpha+\beta}$. Let $\mu_{2}, \mu_{3}$ be the other two eigenvalues. Thus, $-\frac{3 r}{1+\alpha+\beta}=\mu_{1}+\mu_{2}+\mu_{3}=-r+\mu_{2}+\mu_{3}$, and therefore $\mu_{2}+\mu_{3}=$ $-\frac{3 r}{1+\alpha+\beta}+r=\frac{r(\alpha+\beta-2)}{1+\alpha+\beta}<0$, by assumption $\alpha+\beta<2$. Hence, $\mu_{2}+\mu_{3}<0$. On the other hand, $\mu_{1} \mu_{2} \mu_{3}=\operatorname{det} J_{r r r}<0$. Since $\mu_{1}<0$, this implies that $\mu_{2} \mu_{3}>0$. Together with $\mu_{2}+\mu_{3}<0$, this implies that $\operatorname{Re}\left(\mu_{2}\right), \operatorname{Re}\left(\mu_{3}\right)<0$. Thus, all the eigenvalues have negative real parts, which implies asymptotic stability [23].

Now, suppose for some $r_{1}, r_{2}, r_{3}>0$ the fixed point is unstable. Then at least one of the eigenvalues of $J$ has positive real part. By continuous dependence of eigenvalues on matrix entries, there is a point $\left(r_{1}^{*}, r_{2}^{*}, r_{3}^{*}\right)$ at which the real part of at least one eigenvalue becomes zero. Then we either have three real eigenvalues, at least one of which is zero, or one negative real eigenvalue and two purely imaginary eigenvalues. Since $\operatorname{det}\left(J_{r_{1}^{*} r_{2}^{*} r_{3}^{*}}\right)=r_{1}^{*} r_{2}^{*} r_{3}^{*} \operatorname{det}\left(J_{111}\right) \neq 0$, only the second possibility remains: $\mu_{1}<0$, $\mu_{2}=b i$, and $\mu_{3}=-b i$.

The characteristic polynomial of $J_{r_{1}^{*} r_{2}^{*} r_{3}^{*}}$ is given by

$$
\operatorname{det}\left(\mu I-J_{r_{1}^{*} r_{2}^{*} r_{3}^{*}}\right)=\left(\mu-\mu_{1}\right)\left(\mu^{2}+b^{2}\right)=\mu^{3}-\mu_{1} \mu^{2}+b^{2} \mu-\mu_{1} b^{2} .
$$

Expanding the determinant and equating all the coefficients we calculate:

$$
\begin{aligned}
-\mu_{1} & =\frac{1}{1+\alpha+\beta}\left(r_{1}^{*}+r_{2}^{*}+r_{3}^{*}\right), \\
b^{2} & =\frac{1}{(1+\alpha+\beta)^{2}}(1-\alpha \beta)\left(r_{1}^{*} r_{2}^{*}+r_{1}^{*} r_{3}^{*}+r_{2}^{*} r_{3}^{*}\right), \\
-\mu_{1} b^{2} & =\frac{1}{(1+\alpha+\beta)^{3}} r_{1}^{*} r_{2}^{*} r_{3}^{*}\left(1+\alpha^{3}+\beta^{3}-3 \alpha \beta\right) .
\end{aligned}
$$

In particular, we obtain the following equality:

$$
\frac{\left(r_{1}^{*}+r_{2}^{*}+r_{3}^{*}\right)\left(r_{1}^{*} r_{2}^{*}+r_{1}^{*} r_{3}^{*}+r_{2}^{*} r_{3}^{*}\right)}{r_{1}^{*} r_{2}^{*} r_{3}^{*}}=\frac{1+\alpha^{3}+\beta^{3}-3 \alpha \beta}{1-\alpha \beta} .
$$


For the left hand side of this equality, we calculate

$$
\frac{\left(r_{1}^{*}+r_{2}^{*}+r_{3}^{*}\right)\left(r_{1}^{*} r_{2}^{*}+r_{1}^{*} r_{3}^{*}+r_{2}^{*} r_{3}^{*}\right)}{r_{1}^{*} r_{2}^{*} r_{3}^{*}}=3+\frac{r_{1}^{*}}{r_{2}^{*}}+\frac{r_{2}^{*}}{r_{1}^{*}}+\frac{r_{1}^{*}}{r_{3}^{*}}+\frac{r_{3}^{*}}{r_{1}^{*}}+\frac{r_{2}^{*}}{r_{3}^{*}}+\frac{r_{3}^{*}}{r_{2}^{*}} \geq 9
$$

since $x+\frac{1}{x} \geq 2$ for any $x>0$. The condition that the right hand side be greater than or equal to 9 can be written as $\alpha^{3}+\beta^{3}+6 \alpha \beta \geq 8$. However, since $\alpha+\beta<2$, we get

$$
\alpha^{3}+\beta^{3}+6 \alpha \beta<2\left(\alpha^{2}-\alpha \beta+\beta^{2}\right)+6 \alpha \beta=2(\alpha+\beta)^{2}<8,
$$

a contradiction.

Next, we show that when $\lambda$ approaches $\lambda_{c}$ from above, the interior fixed point is stable as well, regardless of whether $\alpha+\beta<2$ is true or not. We make an additional "generic" assumption: when the interior fixed point reaches the boundary of $\mathbb{R}_{+}^{3}$, only one of its coordinates becomes zero. The case when the assumption fails is biologically unlikely.

Proposition 4.10. Suppose $r_{1}, r_{2}, r_{3}>0, \alpha, \beta>0$. Let $\left(u_{1}^{\lambda}, u_{2}^{\lambda}, u_{3}^{\lambda}\right)$ be the interior fixed point of (4.2) where $\lambda<0$. Assume that $\left(u_{1}^{\lambda_{c}}, u_{2}^{\lambda_{c}}, u_{3}^{\lambda_{c}}\right)$ has only one zero component. Then the interior fixed point $\left(u_{1}^{\lambda}, u_{2}^{\lambda}, u_{3}^{\lambda}\right)$ of (4.2) is asymptotically stable for $\lambda \in\left(\lambda_{c}, \lambda_{c}+\varepsilon\right)$ for some $\varepsilon>0$.

Proof. Let $\lambda_{c}<\lambda \leq 0$. The Jacobian of (4.2) at the fixed point $\left(u_{1}^{\lambda}, u_{2}^{\lambda}, u_{3}^{\lambda}\right)$ is given by

$$
J^{\lambda}=\left(\begin{array}{ccc}
-r_{1} u_{1}^{\lambda} & -r_{1} \alpha u_{1}^{\lambda} & -r_{1} \beta u_{1}^{\lambda} \\
-r_{2} \beta u_{2}^{\lambda} & -r_{2} u_{2}^{\lambda} & -r_{2} \alpha u_{2}^{\lambda} \\
-r_{3} \alpha u_{3}^{\lambda} & -r_{3} \beta u_{3}^{\lambda} & -r_{3} u_{3}^{\lambda}
\end{array}\right) .
$$

Using Lemma 4.1, we see that its determinant is negative:

$$
\operatorname{det}\left(J^{\lambda}\right)=-r_{1} r_{2} r_{3} u_{1}^{\lambda} u_{2}^{\lambda} u_{3}^{\lambda} \Delta<0
$$

Without loss of generality, we may assume that $\left(u_{1}^{\lambda}, u_{2}^{\lambda}, u_{3}^{\lambda}\right)$ approaches $\left(u_{1}^{\lambda_{c}}, u_{2}^{\lambda_{c}}, 0\right)$ as $\lambda \rightarrow \lambda_{c}$, with $u_{1}^{\lambda_{c}}, u_{2}^{\lambda_{c}}>0$.

At $\lambda=\lambda_{c}$, the two species subsystem of (4.2) consisting of species 1 and 2 has a stable coexistence outcome $\left(u_{1}^{\lambda_{c}}, u_{2}^{\lambda_{c}}\right)$, since the founder control outcome is not possible for $\lambda>\lambda_{c}$, by the assumption $\alpha \beta<1$ and Remark 3.2. In particular, the eigenvalues of the two-species subsystem at $\left(u_{1}^{\lambda_{c}}, u_{2}^{\lambda_{c}}\right)$ are real and negative.

Now, the eigenvalues of $J^{\lambda_{c}}$ are $\mu_{3}=0$ and the two real negative eigenvalue of the two-species subsystem. Since eigenvalues depend continuously on the matrix entries, and since for $\lambda>\lambda_{c}$, we have $\operatorname{det}\left(J^{\lambda}\right)<0$, we find that all three eigenvalues are real and negative when $\lambda-\lambda_{c}>0$ is small enough. In particular, the interior fixed point is locally asymptotically stable.

In summary, the following scenarios are possible as we decrease $\lambda$ from 0 to $\lambda_{c}$ : 
1. if $\alpha+\beta<2$, the system starts and ends with a stable interior fixed point. Numerical simulations (see Section 6) suggest that it remains stable for intermediate values of $\lambda$;

2. if $\alpha+\beta \geq 2$, the system starts with a stable heteroclinic cycle (and unstable interior fixed point), and ends with a stable interior fixed point; numerical simulations show that the transition occurs via a stable interior limit cycle (namely, the heteroclinic cycle looses its stability before the interior fixed point becomes stable).

For even smaller values, i.e. $\lambda<\lambda_{c}$ the system reduces to a two-species system since either species 1 or species 3 is extinct. The dynamics of such a system were studied numerically in [19] and analytically in [29]. As $\lambda$ decreases, the competitor with the highest growth rate will eventually take over and displace the other species. In particular, for $\lambda<-r_{2}$, species 2 and 3 will be eliminated from the system. For small enough values of $\lambda>-r_{1}$, Theorem 2.1 in [33] implies that $\left(r_{1}, 0,0\right)$ is globally attracting in $\mathbb{R}_{+}^{3}$.

\section{Transitive case}

We now consider the transitive case in which species are ordered with respect to competitive ability, species 1 being the best, species 3 the worst competitor. We order the intrinsic growth rates in the reverse order, i.e. $0<r_{1}<r_{2}<r_{3}$, so that our model is the natural generalization of the two-species competition model in [19].

Again, to reduce the number of parameters we choose the coefficient matrix as

$$
A=\left(\begin{array}{ccc}
r_{1} & r_{1} \beta & r_{1} \beta \\
r_{2} \alpha & r_{2} & r_{2} \beta \\
r_{3} \alpha & r_{3} \alpha & r_{3}
\end{array}\right),
$$

where $0<\beta<1<\alpha$ and $\alpha \beta<1$, as in Case I in the previous section. The first condition ensures the order of competitive ability, the second condition excludes founder control in any of the two-species subsystems by Remark 3.2.

Now, system (4.2) can be rewritten as

$$
\left\{\begin{array}{l}
\frac{d u_{1}}{d t}=r_{1} u_{1}\left[\left(1+\frac{\lambda}{r_{1}}\right)-u_{1}-\beta u_{2}-\beta u_{3}\right. \\
\frac{d u_{2}}{d t}=r_{2} u_{2} \\
\frac{d u_{3}}{d t}=r_{3} u_{3}\left[\begin{array}{l}
\frac{\lambda}{r_{2}} \\
d+\frac{\lambda}{r_{3}}
\end{array}\right)-\alpha u_{1}-u_{2}-\beta u_{3}-\alpha u_{2}-u_{3}
\end{array}\right],
$$

As in the previous section, we begin our analysis with the question of persistence, and we prove some technical lemmas to start with. 
Lemma 5.1. Assume $0<\beta<1<\alpha$ and $\alpha \beta<1$. Then

$\operatorname{det}\left(\begin{array}{ccc}1 & \beta & \beta \\ \alpha & 1 & \beta \\ \alpha & \alpha & 1\end{array}\right)=\alpha \beta(\alpha+\beta-3)+1=\alpha^{2} \beta+\alpha \beta^{2}-3 \alpha \beta+1>0$.

Proof. We consider the determinant as a polynomial in $\alpha$. It is then sufficient to show that its discriminant $\beta^{4}-6 \beta^{3}+9 \beta^{2}-4 \beta$ is negative for all $0<\beta<1$. Dividing the discriminant by $\beta$, we obtain $f(\beta)=\beta^{3}-6 \beta^{2}+9 \beta-4$. We show that $f(\beta)<0$ for all $0<\beta<1$. Note that $f(1)=f^{\prime}(1)=0$, $f^{\prime}(\beta)=3(\beta-1)(\beta-3)$ and $f^{\prime \prime}(1)<0$. Hence $\beta=1$ is a local maximum, and $f(\beta)<0$ for all $\beta<1$, as needed.

Next, we investigate the question of existence of an interior fixed point for (5.1). The following is an easy application of Cramer's rule.

Lemma 5.2. System (5.1) has the unique fixed point $\left(u_{1}^{\lambda}, u_{2}^{\lambda}, u_{3}^{\lambda}\right)$, where

$$
\begin{aligned}
& u_{1}^{\lambda}=\lambda\left(\frac{1-\alpha \beta}{r_{1}}+\frac{\beta(\alpha-1)}{r_{2}}+\frac{\beta(\beta-1)}{r_{3}}\right)+(\beta-1)^{2}, \\
& u_{2}^{\lambda}=\lambda\left(\frac{\alpha(\beta-1)}{r_{1}}+\frac{1-\alpha \beta}{r_{2}}+\frac{\beta(\alpha-1)}{r_{3}}\right)+(\alpha-1)(\beta-1), \\
& u_{3}^{\lambda}=\lambda\left(\frac{\alpha(\alpha-1)}{r_{1}}+\frac{\alpha(\beta-1)}{r_{2}}+\frac{1-\alpha \beta}{r_{3}}\right)+(\alpha-1)^{2} .
\end{aligned}
$$

Note that $u_{2}^{0}=(\alpha-1)(\beta-1)<0$, and thus, there is no interior fixed point for $\lambda=0$. In fact, the exclusion state $(1,0,0)$ is globally stable. As we decrease $\lambda<0$, system (5.1) may admit an interior fixed point and thus become persistent (or even permanent), under certain conditions outlined in the following proposition.

Proposition 5.3. System (5.1) is persistent iff all three of the following inequalities hold:

$$
\begin{aligned}
\lambda\left(\frac{1-\alpha \beta}{r_{1}}+\frac{\beta(\alpha-1)}{r_{2}}+\frac{\beta(\beta-1)}{r_{3}}\right)+(\beta-1)^{2} & >0 \\
\lambda\left(\frac{\alpha(\beta-1)}{r_{1}}+\frac{1-\alpha \beta}{r_{2}}+\frac{\beta(\alpha-1)}{r_{3}}\right)+(\alpha-1)(\beta-1) & >0 \\
\lambda\left(\frac{\alpha(\alpha-1)}{r_{1}}+\frac{\alpha(\beta-1)}{r_{2}}+\frac{1-\alpha \beta}{r_{3}}\right)+(\alpha-1)^{2} & >0 .
\end{aligned}
$$

Proof. The proof is analogous to the proof of Proposition 4.3.

In the following lemma, we show that the coefficient of $\lambda$ in inequality (5.3) is always negative. Therefore, although the inequality fails for $\lambda=0$, it will hold for sufficiently negative $\lambda$. We also show that the coefficient of $\lambda$ in inequality (5.2) is always positive. Therefore, for sufficiently negative $\lambda$, this inequality will be violated. Note that we cannot obtain a similar result regarding inequality (5.4), since the sign of the corresponding coefficient of $\lambda$ depends on the choice of parameters. 
Lemma 5.4. The inequalities

(a) $\frac{\alpha(\beta-1)}{r_{1}}+\frac{1-\alpha \beta}{r_{2}}+\frac{\beta(\alpha-1)}{r_{3}}<0$

(b) $\frac{1-\alpha \beta}{r_{1}}+\frac{\beta(\alpha-1)}{r_{2}}+\frac{\beta(\beta-1)}{r_{3}}>0$

hold for any $\alpha>1>\beta>0$ and $0<r_{1}<r_{2}<r_{3}$.

Proof. (a)

$$
\frac{\alpha(\beta-1)}{r_{1}}+\frac{1-\alpha \beta}{r_{2}}+\frac{\beta(\alpha-1)}{r_{3}}<\frac{\alpha(\beta-1)}{r_{1}}+\frac{1-\alpha \beta}{r_{1}}+\frac{\beta(\alpha-1)}{r_{1}}=\frac{(1-\alpha)(1-\beta)}{r_{1}}<0 .
$$

(b) Similarly with $r_{3}$.

We now define the critical value for (5.3) as

$$
\lambda_{s 2}=\frac{(\alpha-1)(1-\beta)}{\frac{\alpha(\beta-1)}{r_{1}}+\frac{1-\alpha \beta}{r_{2}}+\frac{\beta(\alpha-1)}{r_{3}}} .
$$

Then, by Lemma 5.4(a), $\lambda_{s 2}<0$. Note that (5.3) holds exactly when $\lambda<\lambda_{s 2}$.

Lemma 5.5. Let $\alpha>1>\beta>0,0<r_{1}<r_{2}<r_{3}$. Then inequalities (5.2) and (5.4) are satisfied for $\lambda=\lambda_{s 2}$.

Proof. Substituting $\lambda=\lambda_{s 2}$ into (5.2) we get the condition

$$
\frac{(\alpha-1)(1-\beta)}{\frac{\alpha(\beta-1)}{r_{1}}+\frac{1-\alpha \beta}{r_{2}}+\frac{\beta(\alpha-1)}{r_{3}}}\left(\frac{1-\alpha \beta}{r_{1}}+\frac{\beta(\alpha-1)}{r_{2}}+\frac{\beta(\beta-1)}{r_{3}}\right)+(\beta-1)^{2}>0 .
$$

Since the denominator is negative and $\beta<1$, this is equivalent to

$$
(\alpha-1)\left(\frac{1-\alpha \beta}{r_{1}}+\frac{\beta(\alpha-1)}{r_{2}}+\frac{\beta(\beta-1)}{r_{3}}\right)<(\beta-1)\left(\frac{\alpha(\beta-1)}{r_{1}}+\frac{1-\alpha \beta}{r_{2}}+\frac{\beta(\alpha-1)}{r_{3}}\right) .
$$

After a lot of algebra, this inequality simplifies to

$$
r_{3}\left(r_{1}-r_{2}\right)(\alpha \beta(\alpha+\beta-3)+1)-r_{1} r_{2} \alpha \beta^{2}<0
$$

which holds, since $r_{1}<r_{2}$ and, by Lemma 5.1, $\alpha \beta(\alpha+\beta-3)+1>0$.

Similarly, plugging $\lambda=\lambda_{s 2}$ into (5.4) and simplifying, we eventually get

$$
\left(\alpha \beta^{2}+\alpha^{2} \beta-3 \alpha \beta+1+\alpha\right) r_{1} r_{3}+\left(-\alpha \beta^{2}-\alpha^{2} \beta+3 \alpha \beta-1-\beta\right) r_{1} r_{2}>0 .
$$

This expression can be rewritten as

$$
r_{1}\left(r_{3}-r_{2}\right)(\alpha \beta(\alpha+\beta-3)+1)+r_{1}\left(r_{3} \alpha-r_{2} \beta\right)>0,
$$

which holds by Lemma 5.1 and the assumptions $r_{2}<r_{3}$ and $\alpha>\beta$. 
Now we define the critical value of $\lambda$ for inequalities (5.2) and (5.4) by setting

$$
\lambda_{s 1}=\max \left\{-\frac{(\beta-1)^{2}}{\frac{1-\alpha \beta}{r_{1}}+\frac{\beta(\alpha-1)}{r_{2}}+\frac{\beta(\beta-1)}{r_{3}}},-\frac{(\alpha-1)^{2}}{\frac{\alpha(\alpha-1)}{r_{1}}+\frac{\alpha(\beta-1)}{r_{2}}+\frac{1-\alpha \beta}{r_{3}}}\right\} .
$$

By Lemma 5.4(b), $\lambda_{s 1}<0$ is well-defined. Note that one of the inequalities (5.2) and (5.4) fails exactly when $\lambda<\lambda_{s 1}$. Also, by Lemma 5.5, $\lambda_{s 1}<\lambda_{s 2}$. Note that $\lambda_{s 1}>-r_{1}$, since for $\lambda \leq-r_{1}$ the first species will not persist. In summary:

Proposition 5.6. System (5.1) is persistent exactly when $\lambda_{s 1}<\lambda<\lambda_{s 2}$.

\section{$5.1 \quad$ Heteroclinic cycle}

Next, we investigate under which conditions system (5.1) admits a heteroclinic cycle. In the following lemma, we apply the classification of the two-species system (3.1) to the two-species subsystems of $(5.1)$.

Lemma 5.7. Let $1 \leq i<j \leq 3$ and $\lambda \in\left(-r_{1}, 0\right]$. Then in the two species subsystem consisting of species $i$ and $j$ we have

(a) species $i$ outcompetes $j$ iff $\frac{1+\frac{\lambda}{r_{j}}}{1+\frac{\lambda}{r_{i}}}<\alpha$,

(b) species $i$ coexists with $j$ iff $\alpha<\frac{1+\frac{\lambda}{r_{j}}}{1+\frac{\lambda}{r_{i}}}<\frac{1}{\beta}$,

(c) species $j$ outcompetes $i$ iff $\frac{1+\frac{\lambda}{r_{j}}}{1+\frac{\lambda}{r_{i}}}>\frac{1}{\beta}$.

Note that for $\lambda \in\left(-r_{1}, 0\right)$ we have the inequality

$$
\max \left\{\frac{1+\frac{\lambda}{r_{2}}}{1+\frac{\lambda}{r_{1}}}, \frac{1+\frac{\lambda}{r_{3}}}{1+\frac{\lambda}{r_{2}}}\right\}<\frac{1+\frac{\lambda}{r_{3}}}{1+\frac{\lambda}{r_{1}}} .
$$

Proposition 5.8. Let $\lambda \in\left(-r_{1}, 0\right)$. Then a heteroclinic cycle occurs iff the following inequalities take place

$$
\max \left\{\frac{1+\frac{\lambda}{r_{3}}}{1+\frac{\lambda}{r_{2}}}, \frac{1+\frac{\lambda}{r_{2}}}{1+\frac{\lambda}{r_{1}}}\right\}<\alpha \text { and } \quad \frac{1+\frac{\lambda}{r_{3}}}{1+\frac{\lambda}{r_{1}}}>\frac{1}{\beta} .
$$

Proof. Follows from Lemma 5.7.

We observe that a fraction of the form $\left(1+\lambda / r_{i}\right)\left(1+\lambda / r_{j}\right)^{-1}$ with $r_{i}<r_{j}$, increases as $\lambda$ decreases. Hence, a heteroclinic cycle can occur if the second inequality in (5.8) is satisfied while the first is violated. Therefore, we define the critical values for $\lambda$ that turn the inequalities in (5.8) into equalities. These are

$$
\lambda_{h c 1}=\max \left\{\frac{r_{2} r_{3}(\alpha-1)}{r_{2}-\alpha r_{3}}, \frac{r_{1} r_{2}(\alpha-1)}{r_{1}-\alpha r_{2}}\right\}
$$

and

$$
\lambda_{h c 2}=\frac{r_{1} r_{3}(1-\beta)}{\beta r_{1}-r_{3}}
$$


Note that $\lambda>-r_{1}$ is the necessary and sufficient condition for all three species to persist in the absence of their competitors. The next lemma shows that it is satisfied for $\lambda_{h c 2}$.

Lemma 5.9. $\lambda_{h c 2}>-r_{1}$.

Proof. Suppose $\lambda_{h c 2} \leq-r_{1}$. Then $r_{3}(1-\beta) \geq-\beta r_{1}+r_{3}$ or $\beta\left(r_{1}-r_{3}\right) \geq 0$. The latter contradicts the assumption $r_{1}<r_{3}$.

The necessary condition for a heteroclinic cycle to exist is $\lambda_{h c 1}<\lambda_{h c 2}$. Substituting $\lambda_{h c 2}$ into the first inequality in (5.8) we find the equivalent condition

$$
\max \left\{\frac{\beta r_{1}\left(r_{2}-r_{3}\right)+r_{3}\left(r_{1}-r_{2}\right)}{\beta r_{2}\left(r_{1}-r_{3}\right)}, \frac{r_{2}\left(r_{1}-r_{3}\right)}{\beta r_{1}\left(r_{2}-r_{3}\right)+r_{3}\left(r_{1}-r_{2}\right)}\right\}<\alpha,
$$

Lemma 5.10.

$$
\begin{aligned}
1<\frac{\beta r_{1}\left(r_{2}-r_{3}\right)+r_{3}\left(r_{1}-r_{2}\right)}{\beta r_{2}\left(r_{1}-r_{3}\right)}<\frac{1}{\beta}, \\
1<\frac{r_{2}\left(r_{1}-r_{3}\right)}{\beta r_{1}\left(r_{2}-r_{3}\right)+r_{3}\left(r_{1}-r_{2}\right)}<\frac{1}{\beta} .
\end{aligned}
$$

Proof. First note that for any $\lambda<0$, we have $\left(1+\lambda / r_{i}\right)\left(1+\lambda / r_{j}\right)^{-1}>1$ for $r_{i}>r_{j}$. Hence, this inequality holds also for $\lambda_{h c 2}$. The second part of inequality (5.12) is equivalent to $\beta r_{1}\left(r_{2}-r_{3}\right)>$ $r_{1}\left(r_{2}-r_{3}\right)$, which is equivalent to $\beta<1$. For inequality (5.13), let $x=\frac{r_{2}\left(r_{1}-r_{3}\right)}{\beta r_{1}\left(r_{2}-r_{3}\right)+r_{3}\left(r_{1}-r_{2}\right)}$. Then by the first inequality,

$$
1<\frac{1}{\beta x}<\frac{1}{\beta}
$$

or $1<x<\frac{1}{\beta}$, as needed.

Thus, a heteroclinic cycle exists if we pick parameter $\alpha$ between 1 and $\frac{1}{\beta}$. If a heteroclinic cycle exists, we can use Theorem 3.3 to determine its stability. If the cycle is attractive then the system is not permanent. The condition for attractiveness of the heteroclinic cycle takes the form

$$
\begin{aligned}
& \left(\alpha r_{3}\left(r_{1}+\lambda\right)-r_{1}\left(r_{3}+\lambda\right)\right)\left(\beta r_{1}\left(r_{2}+\lambda\right)-r_{2}\left(r_{1}+\lambda\right)\right)\left(\beta r_{2}\left(r_{3}+\lambda\right)-r_{3}\left(r_{2}+\lambda\right)\right)> \\
& \left(r_{1}\left(r_{2}+\lambda\right)-\alpha r_{2}\left(r_{1}+\lambda\right)\right)\left(r_{2}\left(r_{3}+\lambda\right)-\alpha r_{3}\left(r_{2}+\lambda\right)\right)\left(r_{3}\left(r_{1}+\lambda\right)-\beta r_{1}\left(r_{3}+\lambda\right)\right) .
\end{aligned}
$$

Summarizing our results, we obtain the following proposition.

Proposition 5.11. Given $0<r_{1}<r_{2}<r_{3}, 0<\beta<1<\alpha$, such that $\alpha \beta<1$, then system (5.1) is persistent exactly when $\lambda_{s 1}<\lambda<\lambda_{s 2}$. In addition, if inequality (5.11) holds, then

(a) $\lambda_{s 1} \leq \lambda_{h c 1}<\lambda_{h c 2} \leq \lambda_{s 2}$;

(b) System (5.1) admits a heteroclinic cycle for $\lambda_{h c 1}<\lambda<\lambda_{h c 2}$;

(c) If (5.14) holds, then the heteroclinic cycle is attractive. 
Figure 2 illustrates the above proposition. Depending on parameters, we may have a subinterval of $\left(\lambda_{h c 1}, \lambda_{h c 2}\right)$ where any solution will approach a stable heteroclinic cycle. If the inequality above fails for $\lambda \in\left(\lambda_{h c 1}, \lambda_{h c 2}\right)$, then the heteroclinic cycle is unstable and therefore the system is permanent; i.e. we have stable coexistence of all three species.

\section{$6 \quad$ Numerical Results}

In this section, we illustrate the results from the previous two sections, and we thereby classify the possible effects of changes in flow speed on the outcome of competition of three species in advective environments. In each subsection, we begin with the effect of decreasing $\lambda$ in the spatially implicit models (4.2) and (5.1), and then address the comparison with increasing flow speed $q$ in the corresponding spatial model (2.1). Recall that $\lambda=\lambda(d, q)$ is a decreasing function of $q$. We use triangular diagrams to represent the relation between competitors and location of a fixed point. An arrow between species $i$ and $j$ points into the direction of the superior species. A square indicates the stable equilibrium of the full model. Its location indicates whether it is an interior coexistence point (meaning permanence), or loss of persistence of the whole system if one or two species are excluded. A summary of the qualitative behavior and specific parameter values is given in Tables 1 and 2 .

\subsection{Cyclic case I}

We choose parameters $\alpha>1>\beta>0$, so that for $\lambda=0$, species $i$ beats species $i-1$ (modulo 3 ). As in section 4 , we choose $r_{1}>r_{2}>r_{3}$ and $\alpha \beta<1$. Founder control is excluded from any of the two-species sub-systems, according to Remark 3.2. A number of different sub-cases is possible.

Cyclic permanent case I. For $\lambda=0$, the spatially implicit system (4.2) is permanent iff $\alpha+\beta<$ 2. The interior fixed point $(\gamma, \gamma, \gamma)$ is stable, according to Proposition 4.9. The cyclic arrangement of species is shown in Figures 4 (a) and 8 (a). As we decrease $\lambda$, coexistence fails if one of the inequalities (4.3) or (4.5) is violated.

Cyclic permanent case I (a): If inequality (4.5) becomes violated at $\lambda_{c}=\lambda_{c 2}>\lambda_{c 1}$, then species 3 disappears while species 1 and 2 coexist. For example, choosing $r_{1}=1.8, r_{2}=1.3, r_{3}=$ $1, \alpha=1.5, \beta=0.4$, we calculate $\lambda_{c}=\max \left(\lambda_{c 1}, \lambda_{c 2}\right)=\max (-0.8738,-0.8437)=-0.8437$. Once species 3 disappears, we observe the competition between a competitively superior species 2 , and an inferior species 1 , which has the higher growth rate. As we decrease $\lambda$ further, species 1 gradually replaces species 2, as predicted in [19, 29]. This sequence of events is summarized in Figure 4.

The spatial model goes through the same stages as we increase advection. The coexistence of three species is replaced by coexistence of the first and second species, with the first species winning under sufficiently high advection, see Figure 6 . 
Cyclic permanent case I (b): If inequality (4.3) is violated for $\lambda_{c}=\lambda_{c 1}>\lambda_{2}$, then species 1 disappears while species 2 and 3 coexist. This happens, for example, for $r_{1}=1.6, r_{2}=1.3, r_{3}=$ $1, \alpha=1.5, \beta=0.4$, where we calculate $\left(\lambda_{c 1}, \lambda_{c 2}\right)=(-0.8511,-0.9233)$. Figure 8 summarizes the effect of decreasing $\lambda$ : coexistence of species 2 and 3 (triangle (b)) is replaced by domination of species 2 (triangle (c)), then coexistence of species 1 and 2 (triangle (d)), with an eventual domination by species 1 (triangle (e)).

In this case, we observe that the explicitly spatial model goes through qualitatively similar stages with one exception. Unlike in the implicit case, species 1 does not go to extinction for intermediate values of advection. It does persist, however, only at very low density, see Figure 10. Hence, we get a first example that the implicit model cannot capture all of the spatially explicit model behavior.

Non-permanent Cyclic case I: For $\lambda=0$, the spatially implicit system (4.2) is non-permanent iff $\alpha+\beta \geq 2$ (Figure 12, triangle (a)). The interior fixed point it unstable, solutions approach the heteroclinic cycle, see Theorem 16.1.1 in [13]. This situation occurs, for example, for $r_{1}=1.6$, $r_{2}=1.3, r_{3}=1, \alpha=1.6, \beta=0.5$. The heteroclinic cycle is attractive for $-0.66<\lambda<0$. For $-0.68<\lambda<-0.66$, we numerically observe a limit cycle behavior: all three species oscillate above the zero density (triangle (b)). The heteroclinic cycle and the interior fixed point are both unstable. As we decrease $\lambda$ even further, the oscillations eventually stabilize at the interior fixed point (triangle (c)). When $\lambda$ is increased even further, the behavior of the model follows one of the two permanent cases described above ((a) and (b)).

The explicit spatial model undergoes the same qualitative changes as we increase advection. For small values of $q$, we observe a heteroclinic cycle, e.g. $q=0.8, L=10$, see Figure 14 . For increased advection $(q=0.87)$ one observes periodic solutions, see Figure 16.

\subsection{Cyclic case II}

We choose parameters $\beta>1>\alpha>0$, so that for $\lambda=0$, species $i$ beats $i+1$ (modulo 3 ). Again, we distinguish several cases.

Cyclic permanent case II. For $\lambda=0$, the interior fixed point $(\gamma, \gamma, \gamma)$ is stable, see Proposition 4.9. This corresponds to triangle (a), Figure 18. Example parameter values are $r_{1}=1.6, r_{2}=1.3, r_{3}=$ 1 , and $\alpha=0.4, \beta=1.5$. As we decrease $\lambda<0$, coexistence fails when inequality (4.4) is violated. Species 2 goes extinct for $\lambda_{c}=-0.68$, whereas species 1 and 3 coexist (triangle (b)). Further decrease in $\lambda$ eventually leads to extinction of species 1 (triangle (c)). Note that, contrary to Figure 4 , the arrows on the subsystem involving the extinct species do not change. We had mentioned before that the direction of the arrows can change with advection only if the growth rate of the weaker species is higher; this is not the case here. 
Simulations of the spatially explicit model are fully in line with the behavior of the implicit model. With the same values as above $(d=1, L=10)$, we observe that the second species goes extinct for advection speeds of $q \approx 1.4$ or greater. Then we are in the two-species competition case, and for high enough advection, the species with the highest growth rate will win [19].

Non-permanent Cyclic case II: This case is essentially the same as the Non-permanent Cyclic case I with arrows reversed; we omit the details.

\subsection{Transitive case}

For $\lambda=0$, the system is non-persistent, as the superior competitor (species 1) excludes the other two. The system becomes persistent for $\lambda_{s 1}<\lambda<\lambda_{s 2}$. Then it loses persistence, and the competitor with the highest intrinsic growth rate dominates (species 3). Depending on the existence and stability of a heteroclinic cycle within the persistence interval, three different cases are possible: no heteroclinic cycle, unstable heteroclinic cycle, and stable heteroclinic cycle.

Transitive case (a): no heteroclinic cycle. In the first case, the coexistence equilibrium is attractive whenever it exists. For example, if we choose $r_{1}=1, r_{2}=1.2, r_{3}=1.5, \alpha=1.2, \beta=0.5$, then we can calculate $\lambda_{s 1}=-0.7895, \lambda_{s 2}=-0.5, \lambda_{h c 1}=-0.5455$, and $\lambda_{h c 2}=-0.75$. In particular, the heteroclinic cycle is never admissible since $\lambda_{h c 1}>\lambda_{h c 2}$. The system is permanent throughout the persistence interval $\lambda_{s 1}<\lambda<\lambda_{s 2}$. The qualitative changes in the system for decreasing $-1.1<\lambda<0$ are depicted in Figure 20.

Transitive case (b): unstable heteroclinic cycle. In this case, inside the persistence interval there is an interval where the heteroclinic cycle is admissible, but it is never an attractor. For example, let $r_{1}=1, r_{2}=1.4, r_{3}=2.2, \alpha=1.7, \beta=0.5$. Then we calculate $\lambda_{s 1}=-0.7526, \lambda_{h c 1}=-0.7101$, $\lambda_{h c 2}=-0.6471, \lambda_{s 2}=-0.5996$. The heteroclinic cycle exists for $\lambda_{h c 1}<\lambda<\lambda_{h c 2}$ but is unstable since inequality (5.14) fails. Within this case, there are still a number of different qualitative behaviors (analogous to cyclic case (a) and (b)), depending on the exact choice of parameters, see Figure 22.

Transitive case (c): stable heteroclinic cycle. In this case, there is a range of values of $\lambda$ for which the heteroclinic cycle is attractive. For example, if we choose $r_{1}=1, r_{2}=1.5, r_{3}=2.7, \alpha=2$, $\beta=0.49$, then we calculate the threshold value as $\lambda_{s 1}=-0.7533, \lambda_{h c 1}=-0.75, \lambda_{h c 2}=-0.6231$, $\lambda_{s 2}=-0.618$. The heteroclinic cycle exists for $\lambda_{h c 1}<\lambda<\lambda_{h c 2}$. Moreover, for $-0.76<\lambda<-0.63$, inequality (5.14) holds, and thus the heteroclinic cycle is stable. The sequence of qualitative behaviors in this case is very similar to Figure 22, except that in the top row, two triangles would be added: a stable heteroclinic cycle (triangle (e)) and a second copy of triangle (d) after that (triangle (f)), see Figure 24 .

In all three cases, we compared the qualitative behavior of the spatially implicit model with the 
spatially explicit model. We found good agreement in all cases except the stable heteroclinic cycle (see Discussion). In particular, we found a direct generalization of the results in [19, 29]: advection can facilitate coexistence between three competing species that would otherwise not coexist, provided that the weakest competitor has the highest intrinsic growth rate.

\subsection{Comparison of implicit and explicit spatial models}

The goal of replacing the spatially explicit model with a spatially implicit model is to gain insight into the qualitative behavior of its solutions from analyzing a much simpler model. As is evident from the results reported above, the spatially explicit model shows much the same behavior for increasing $q$ as the implicit model for decreasing $\lambda$. A natural question is then to ask how well the critical values of $\lambda$ predict the critical values of $q$, or, in other words, how well we can predict the bifurcation points. We focus our discussion on the persistence criteria.

In the cyclic permanent cases I and II (Subsections 4.1, 4.2) persistence of the three species is lost at $\lambda=\lambda_{c}$. In the spatially explicit system, persistence is lost at some critical value $q_{c}$. We compared $\lambda_{c}$ with $\lambda\left(d, q_{c}\right)$ as described in the past paragraph or section 2. We chose $L=10$ and $d=1$.

In the Cyclic permanent case I (a), we found numerically that the third species disappears for $q=q_{c} \approx 1.53$. The corresponding principal eigenvalue is $\lambda_{1}\left(d, q_{c}\right) \approx-0.6482>\lambda_{c}=-0.8437$.

In the Cyclic permanent case I (b), species 1 never completely disappears in the spatially explicit model, whereas it does in the implicit model. However, for $q=1.62$, the density of species 1 is extremely small compared to the other two species. The corresponding eigenvalue is $\lambda_{1}(1,1.62) \approx$ $-0.7287>\lambda_{c}=-0.8511$.

In the Cyclic permanent case II, we notice that the second species goes extinct for $q=q_{c} \approx 1.38$. The corresponding eigenvalue is $\lambda_{1}\left(1, q_{c}\right) \approx-0.5366>\lambda_{c}=-0.69$, see Table 2 for a summary of these values.

In the transitive case, increasing advection in the spatially explicit model, we obtain persistence of the three species in some interval $q_{1}<q<q_{2}$. Within this interval, there may or may not be a (stable) heteroclinic cycle, see cases (a), (b), or (c) above. Numerically, we found persistence in the intervals $1.09<q<1.55$ (case (a)), $1.22<q<1.53$ (case (b)), and $1.25<q<1.51$ (case (c)). These values of $q$ correspond to principal eigenvalues $\lambda_{1}$ in the interval $(-0.6639,-0.3515)$ (case (a)), $(-0.6482,-0.4295)$ (case (b)), and $(-0.6327,-0.4486)$ (case (c)). For the spatially implicit model, we obtained persistence for $-0.7895<\lambda<-0.5$ (case (a)), $-0.7526<\lambda<-0.5996$ (case (b)), and $-0.7533<\lambda<-0.618$ (case (c)), see Table 2 for a summary of these values.

In all cases that we checked, we found that if $q^{*}$ is a bifurcation point for the explicit model and 
Table 1: Summary of dynamic behavior and comparison of parameter values for the cyclic permanent cases.

\begin{tabular}{|c|c|c|c|}
\hline & Cyc. Perm. Case Ia & Cyc. Perm. Case Ib & Cyc. Perm. Case II \\
\hline$\left(r_{1}, r_{2}, r_{3}\right)$ & $(1.8,1.3,1)$ & $(1.6,1.3,1)$ & $(1.6,1.3,1)$ \\
$(\alpha, \beta)$ & $(1.5,0.4)$ & $(1.5,0.4)$ & $(0.4,1.5)$ \\
$\lambda_{c 1}$ & -0.8738 & -0.8511 & $\mathrm{n} / \mathrm{a}$ \\
$\lambda_{c 2}$ & -0.8437 & -0.9233 & $\mathrm{n} / \mathrm{a}$ \\
& species 3 extinct at: & species 1 extinct at: & species 2 extinct at: \\
$\lambda_{c}$ & $\max \left(\lambda_{c 1}, \lambda_{c 2}\right)=-0.8437$ & $\max \left(\lambda_{c 1}, \lambda_{c 2}\right)=-0.8511$ & -0.68 \\
$q_{c}$ & 1.53 & 1.62 & 1.38 \\
$\lambda_{1}\left(1, q_{c}\right)$ & -0.6482 & -0.7287 & -0.5366 \\
\hline
\end{tabular}

Table 2: Summary of dynamic behavior and comparison of parameter values for the transitive case.

\begin{tabular}{|c|c|c|c|}
\hline & Trans. Case a & Trans. Case b & Trans. Case c \\
\hline$\left(r_{1}, r_{2}, r_{3}\right)$ & $(1,1.2,1.5)$ & $(1,1.4,2.2)$ & $(1,1.5,2.7)$ \\
$(\alpha, \beta)$ & $(1.2,0.5)$ & $(1.7,0.5)$ & $(2,0.49)$ \\
$\lambda$-interval of persistence & & & \\
$\left(\lambda_{s 1}, \lambda_{s 2}\right)$ & $(-0.7895,-0.5)$ & $(-0.7526,-0.5996)$ & $(-0.7533,-0.618)$, \\
$\lambda$-interval of heteroclinic & & & \\
$\left(\lambda_{h c 1}, \lambda_{h c 2}\right)$ & $\emptyset$ & $\emptyset$ & $(-0.75,-0.6231)$ \\
heteroclinic stability interval & $n / \mathrm{a}$ & $(-0.76,-0.63)$ \\
$q$-interval of persistence & & $(1.22,1.53)$ & $(1.25,1.51)$ \\
$\left(q_{1}, q_{2}\right)$ & $(1.09,1.55)$ & $(-0.6482,-0.4295)$ & $(-0.6327,-0.4486)$ \\
\hline
\end{tabular}


$\lambda^{*}$ is the corresponding bifurcation point in the implicit model, then $\lambda\left(q^{*}\right)>\lambda^{*}$. In particular, the implicit model seems to always overestimate the value of $q$ at the bifurcation point.

\section{Conclusions and Biological implications}

River ecosystems provide essential services to humans (e.g. fresh water, transportation, recreation), and are at the same time altered and frequently stressed by many human activities (e.g. water extraction, flow regulation, pollution). For example, increasing water demands in Southern Alberta, Canada, stem from industrial use (e.g. oil extraction), farm requirements, household demand, and recreational requirements (e.g. golf courses) in an area that has suffered extensive drought periods in past decades. In this work, we explored how changes in water discharge may affect community composition in rivers by looking at the dynamics of three competing species in an advective environment with changing flow speed.

We found that changes in flow speed can fundamentally change species composition. Whereas competitive ability determines the outcome of competition at relatively low speeds, intrinsic growth rates become more important, and, in fact, the deciding factor at high flow speeds. In particular, our results predict that if continued water extraction leads to decreased flow speeds, new species could invade and coexist or even replace existing species in such rivers. There is at least anecdotal evidence that such invasion and replacement occurs in some rivers in Southern Alberta, where blue-green algae seem to replace diatoms (Ed McCauley, personal communication). Another biological result of our analysis relates to the various forms in which three species can stably coexist in the transitive case. If advection is such that there is an unstable heteroclinic cycle (see e.g. Figure 22, Transitive Case (b)), then the removal of one species would necessarily imply the extinction of another species, whereas if there is no heteroclinic cycle (either due to different flow speed as in Figure 22, or due to different interaction parameters as in Figure 20) then after the removal of one species, the other two can coexist.

Our major tool to study these changes was to reduce the reaction-advection-diffusion system to an ordinary differential equation system by replacing the spatial differential operator with its dominant eigenvalue. Our reason for this simplification is that while stability conditions for these reactiondiffusion systems can be written down formally in terms of eigenvalues and variational equations $[3,7]$, the steady states and corresponding eigenvalue problems have to be solved numerically in the end. More importantly, while the principle that 'mutual invasion implies stable coexistence' holds for two competing species, the dynamics of three competing species is much richer. Even if all species can persist at low density, it is not clear whether the outcome is stable coexistence, periodic coexistence, or a heteroclinic cycle.

We analyzed the resulting 3 -species competition system in detail, and then compared the results 
to numerical simulations of the reaction-diffusion system. We found a very good agreement of the qualitative changes in the system in most cases. In some very vague sense, the implicitly spatial model can be considered as a model for the spatial averages only. Given this crude "approximation", it is surprising how well the implicit model predicts the behavior of the spatial model. While we are not aware of any formal approximation results, other authors have also successfully used this idea to predict the behavior of predator-prey interaction in fragmented habitats [28], and for population persistence and cycles in an integrodifference equation [18]. Of course, the persistence condition of a single species is determined precisely by the dominant eigenvalue [7].

There are, of course, cases where the implicit model does not accurately predict the behavior of the explicit model. The most interesting is the question of a heteroclinic cycle in the transitive case. We did find a heteroclinic cycle numerically in the reaction-advection-diffusion system, but only when $\alpha \beta>1$, i.e. when the competition coefficients were such that the two-species subsystems showed founder control outcomes in the absence of advection and diffusion, see Figure 26. There are other cases, where the implicit model cannot ever accurately predict the behavior of the explicit model, for example the question of pattern formation [15].

A number of questions arise from our work. First of all, we would like to have some measure for how good this spatially implicit "approximation" is and under what conditions. Elsewhere, we numerically compared the spatial average of the explicit model with the implicit model for a two-species model, and found reasonably good agreement [29], but again, there are no estimates available. Similarly, it would be nice to know whether the observation $\lambda\left(q^{*}\right)>\lambda^{*}$ from the end of the previous section always holds. In particular, if the methods of $[9,8]$ can be extended to our system with advection, then the question of permanence could be evaluated and compared with the implicit system.

Much more biologically relevant is the question of what happens when the three species experience different advection and diffusion rates. We consider our work as a "proof of concept" to show that advection can alter competitive outcome and serve as a coexistence mechanism. Of course, if we allow the three species to vary in their advection and diffusion rates, even more opportunities for coexistence will appear. In such an enlarged parameter space, it is easy to imagine trade-offs between competitive ability and drift or diffusion capability. But also qualitatively new phenomena appear, for example pattern formation [15]. As a final remark, we mention that our results on the implicit system remain true for other interpretations of $\lambda$. In particular, if $\lambda$ is considered as a harvesting rate, then our results show that uniform (as opposed to species specific) harvesting can lead to or destroy coexistence of three competing species. 


\section{References}

[1] Mary Ballyk, Le Dung, Don A. Jones, and Hal Smith. Effects of random motility on microbial growth and competition in a flow reactor. SIAM J. Appl. Math., 59(2):573-596, 1998.

[2] Mary Ballyk and Hal Smith. A flow reactor with wall growth. In Mary Ann Horn, editor, Mathematical models in medical and health science, pages 17-28. Nashville, Vanderbilt University Press, 1998.

[3] Mary Ballyk and Hal Smith. A model of microbial growth in a plug flow reactor with wall attachment. Math. Biosci., 158:95-126, 1999.

[4] H. Berestycki, O. Diekmann, C.J. Nagelkerke, and P.A. Zegeling. Can a species keep pace with a shifting climate? Bull. Math. Biol., 71(2):399-429, 2009.

[5] B. Boldin. Persistence and spread of gastro-intestinal infections: the case of enterotoxigenic escherichia coli in piglets. Bull. Math. Biol., 70(7):2077-2101, 2007.

[6] B. Bolker and S. Pacala. Spatial moment equations for plant competition: understanding the advantages of local dispersal. Am. Nat., 153(6):575-602, 1999.

[7] R. S. Cantrell and C. Cosner. Spatial Ecology via Reaction-Diffusion Equations. Mathematical and Computational Biology. Wiley, 2003.

[8] R.S. Cantrell, C. Cosner, and V. Hutson. Permanence in ecological systems with spatial heterogeneity. Proc. Royal Soc. Edinburgh, 123A(533-559), 1993.

[9] R.S. Cantrell, C. Cosner, and V. Hutson. Permanence in some diffusive Lotka-Volterra models for three interacting species. Dyn. Sys. Appl., 2:505-530, 1993.

[10] P. Chesson. General theory of competitive coexistence in spatially-varying environments. Theor. Pop. Biol., 58:211-237, 2000.

[11] C.-W. Chi, S.-B. Hsu, and L.-I. Wu. On the asymmetric may-leonard model of three competing species. SIAM J. Appl. Math, 58(1):211-226, 1998.

[12] J.P. Grover, S.-B. Hsu, and F.-B. Wang. Competition and coexistence in flowing habitats with a hydraulic storage zone. Math. Biosci., 222(42-52), 2009.

[13] J. Hofbauer and K. Sigmund. Evolutionary games and population dynamics. Cambridge University Press, 1998. 
[14] J. Huisman, M. Arrayás, U. Ebert, and B. Sommeijer. How do sinking phytoplankton species manage to persist. Am. Nat., 159:245-254, 2002.

[15] K. Kishimoto. The diffusive Lotka-Volterra system with three species can have a sable nonconstant equilibrium solution. J. Math. Biol., 16:103-112, 1982.

[16] M. Kot. Elements of Mathematical Ecology. Cambridge Univ. Press, Cambridge, 2001.

[17] C.L. Lehmann and D. Tilman. Competition in spatial habitats. In D. Tilman and P. Kareiva, editors, Spatial Ecology, volume 30 of Monographs in Population Biology, pages 185-203. Princeton University Press, 1997.

[18] F. Lutscher and M. A. Lewis. Spatially-explicit matrix models. A mathematical analysis of stagestructured integrodifference equations. J. Math. Biol., 48:293-324, 2004.

[19] F. Lutscher, E. McCauley, and M.A. Lewis. Spatial patterns and coexistence mechanisms in rivers. Theor. Pop. Biol., 71(3):267-277, 2007.

[20] F. Lutscher, E. Pachepsky, and M.A. Lewis. The effect of dispersal patterns on stream populations. SIAM REV., 47(4):749-772, 2005.

[21] R.M. May and J. Leonard. Nonlinear aspects of competition between three species. SIAM J. Appl. Math, 29:243-253, 1975.

[22] E. Pachepsky, F. Lutscher, R. Nisbet, and M. A. Lewis. Persistence, spread and the drift paradox. Theor. Pop. Biol., 67:61-73, 2005.

[23] L. Perko. Differential equations and dynamical systems. Springer, New York, 2000.

[24] A.B. Potapov and M.A. Lewis. Climate and competition: The effect of moving range boundaries on habitat invasibility. Bull. Math. Biol., 66(5):975-1008, 2004.

[25] J.M. Pringle, F. Lutscher, and E. Glick. Going against the flow: the effect of non-gaussian dispersal kernels and reproduction over multiple generations. Marine Ecol. Prog. Ser., 337:13-17, 2009.

[26] J. Sherratt. An analysis of vegetation stripe formation in semi-arid landscapes. J. Math. Biol., 51:183-197, 2005.

[27] D.C. Speirs and W.S.C. Gurney. Population persistence in rivers and estuaries. Ecology, 82(5):1219-1237, 2001. 
[28] S. Strohm and R. Tyson. The effect of habitat fragmentation on cyclic population dynamics: A reduction to ordinary differential equations. Theor. Ecol., 2011.

[29] O. Vasilyeva. Modeling and Analysis of Population dynamics in advective environments. PhD thesis, University of Ottawa, 2011.

[30] E.C. Zeeman and M.L. Zeeman. An n-dimensional Lotka-Volterra system is generically determined by the edges of its carrying simplex. Nonlinearity, 15:2019-2032, 2002.

[31] E.C. Zeeman and M.L. Zeeman. From local to global behavior in competitive Lotka-Volterra systems. Trans. Amer. Math. Soc., 355:713-734, 2003.

[32] M.L. Zeeman. Hopf bifurcations in competitive three-dimensional Lotka-Volterra systems. Dynamics Stability Systems, 8:189-217, 1993.

[33] M.L. Zeeman. Extinction in competitive Lotka-Volterra systems. Proc. Amer. Math. Soc., 123(1):87-96, 1995.

\section{Figures}

Figure 2: Persistence interval for the transitive case as summarized in Proposition 5.11.

Figure 4: Effect of advection on competition in the Cyclic permanent case I (a). Here and in all similar Figures, advection increases from left to right.

Figure 6: Steady states of the spatially explicit model in the Cyclic permanent case I (a). Left: All three species stably coexist in the habitat. Right: The third species went extinct. The plot on the left (right) corresponds to the first (second) plot in Figure 4. Parameter values are $\alpha=1.5, \beta=0.4, r_{1}=$ $1.8, r_{2}=1.3, r_{3}=1, d=1, L=10$ and advection $q=1.3$ (left plot) and $q=1.6$ (right plot).

Figure 8: Effect of advection on competition in the Cyclic Permanent case I (b).

Figure 10: The first species is almost extinct for the spatial model in the Cyclic permanent case I (b). According to the spatially implicit approximation, the first species should be extinct, see the second triangle in Figure 8. Parameter values are $r_{1}=1.6, r_{2}=1.3, r_{3}=1, \alpha=1.5, \beta=0.4, d=1, L=10$, and advection $q=1.62$. 
Figure 12: Effect of advection on competition in the Non-permanent Cyclic case I.

Figure 14: The spatially explicit model shows an attractive heteroclinic cycle in the Non-permanent Cyclic case I. Left: density of species 1 as a function of space and time. Right: Contour plot of the density of species 1, where dark (light) represents low (high) density. We can clearly see how the intervals of high and low density become longer over time. Parameter values are $q=0.8, r_{1}=1.6$, $r_{2}=1.3, r_{3}=1, \alpha=1.6, \beta=0.5, L=10, d=1$.

Figure 16: The spatially explicit model shows an attractive periodic orbit in the Non-permanent Cyclic case I. Left: density of species 1 as a function of space and time. Right: Contour plot of the density of species 1, where dark (light) represents low (high) density. In contrast to the previous figure, we can clearly see how the intervals of high and low density alternate periodically. Parameter values are $q=0.87$, and the rest as in the previous figure.

Figure 18: Effect of advection on competition in the Permanent Cyclic case II. As advection increases, the second species is the first to disappear.

Figure 20: Effect of advection on competition in the Transitive case (a). The stable coexistence state of all three species is depicted in triangle (c). Of course, not both of the subsystems involving species 2 need to have stable coexistence at the same time. The stable interior coexistence state exists for some other combinations as well (not shown).

Figure 22: Effect of advection on competition in the Transitive case (b). Stages in brackets may or may not occur for different choices of parameters.

Figure 24: Effect of advection on competition in the Transitive case (c). The major difference to the previous plot is that the heterogeneous cycle is now stable for intermediate values of $\lambda$, see triangle (e).

Figure 26: The spatially explicit model shows an attractive heteroclinic cycle in the transitive case with $\alpha \beta>1$. Left: density of species 1 as a function of space and time. Right: Contour plot of the density of species 1, where dark (light) represents low (high) density. In contrast to the previous figure, we can clearly see how the intervals of high and low density alternate periodically. Parameter values are $q=0.8, r_{1}=1, r_{2}=1.1, r_{3}=1.4, \alpha=0.69, \beta=1.5, L=10, d=1$. 


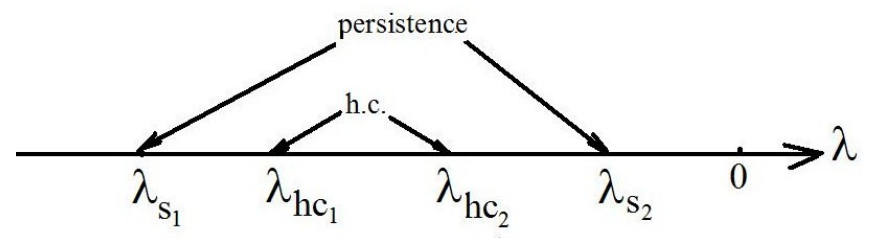

Figure 1: Persistence interval for the transitive case as summarized in Proposition 5.11.

Figure 2:
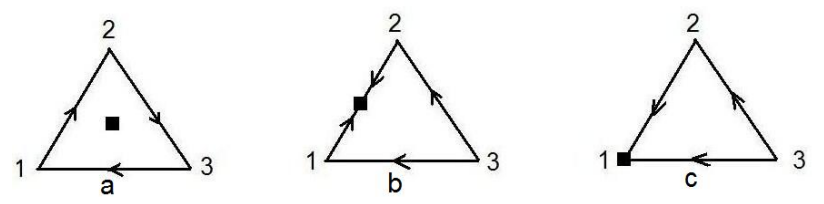

Figure 3: Effect of advection on competition in the Cyclic permanent case I (a). Here and in all similar Figures, advection increases from left to right.

Figure 4:
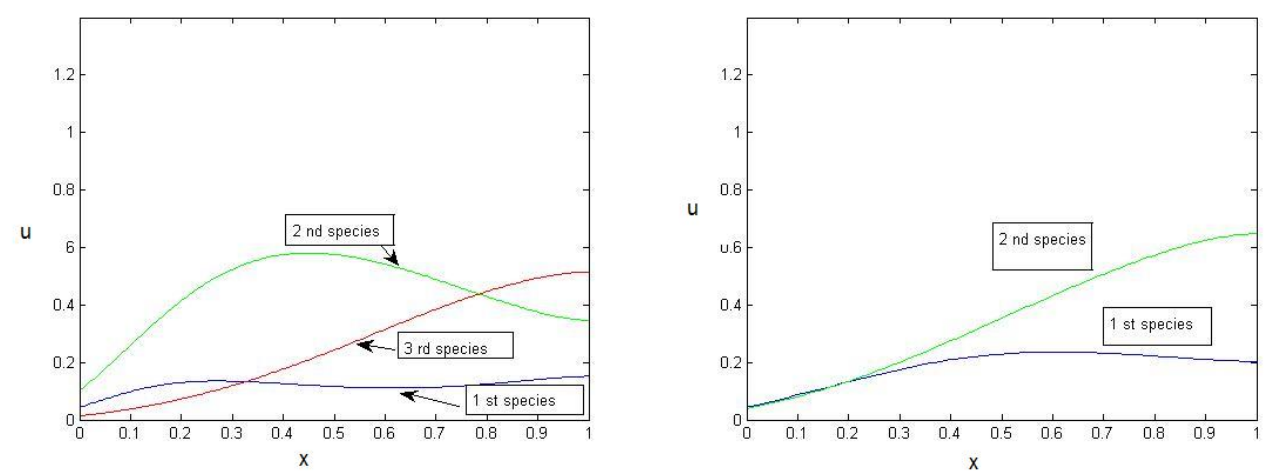

Figure 5: Steady states of the spatially explicit model in the Cyclic permanent case I (a). Left: All three species stably coexist in the habitat. Right: The third species went extinct. The plot on the left (right) corresponds to the first (second) plot in Figure 4. Parameter values are $\alpha=1.5, \beta=0.4, r_{1}=$ $1.8, r_{2}=1.3, r_{3}=1, d=1, L=10$ and advection $q=1.3$ (left plot) and $q=1.6$ (right plot).

Figure 6: 


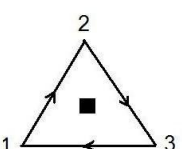

a

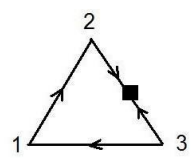

b

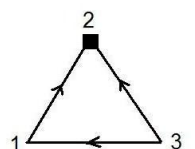

c

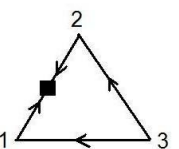

d

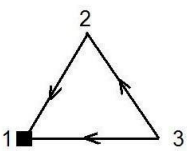

e

Figure 7: Effect of advection on competition in the Cyclic Permanent case I (b).

Figure 8:

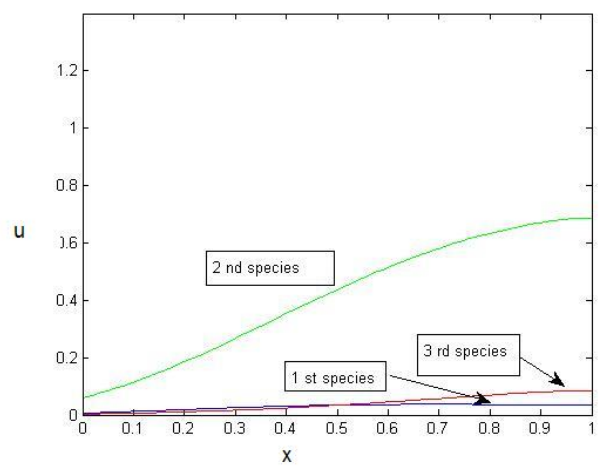

Figure 9: The first species is almost extinct for the spatial model in the Cyclic permanent case I (b). According to the spatially implicit approximation, the first species should be extinct, see the second triangle in Figure 8. Parameter values are $r_{1}=1.6, r_{2}=1.3, r_{3}=1, \alpha=1.5, \beta=0.4, d=1, L=10$, and advection $q=1.62$.

Figure 10:
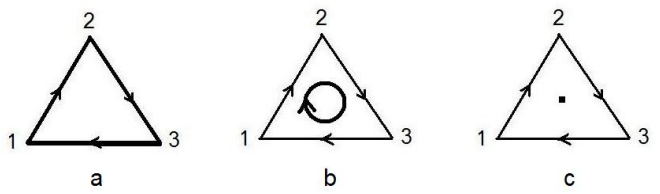

Figure 11: Effect of advection on competition in the Non-permanent Cyclic case I.

Figure 12: 

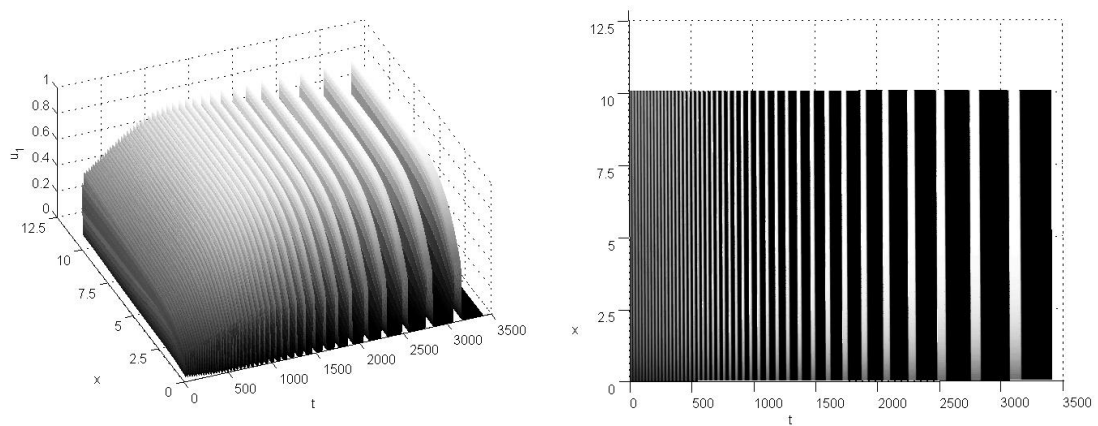

Figure 13: The spatially explicit model shows an attractive heteroclinic cycle in the Non-permanent Cyclic case I. Left: density of species 1 as a function of space and time. Right: Contour plot of the density of species 1, where dark (light) represents low (high) density. We can clearly see how the intervals of high and low density become longer over time. Parameter values are $q=0.8$, $r_{1}=1.6, r_{2}=1.3, r_{3}=1, \alpha=1.6, \beta=0.5, L=10, d=1$.

Figure 14:
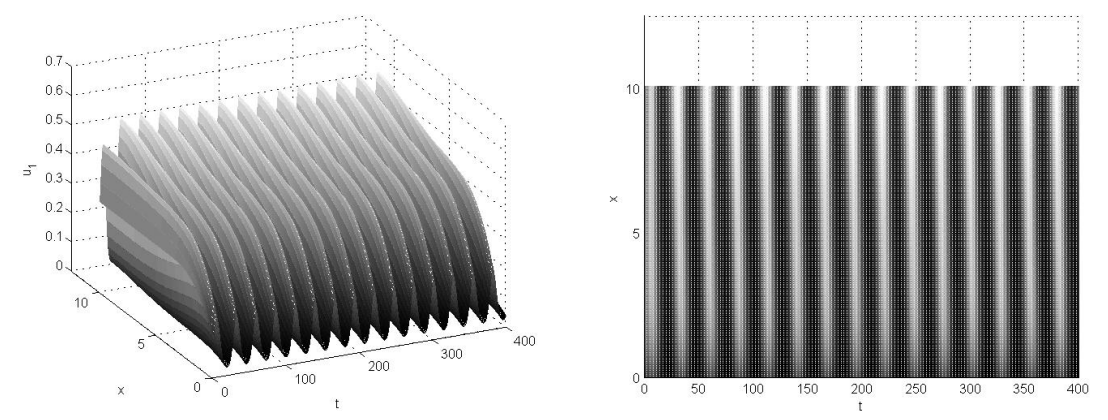

Figure 15: The spatially explicit model shows an attractive periodic orbit in the Non-permanent Cyclic case I. Left: density of species 1 as a function of space and time. Right: Contour plot of the density of species 1, where dark (light) represents low (high) density. In contrast to the previous figure, we can clearly see how the intervals of high and low density alternate periodically. Parameter values are $q=0.87$, and the rest as in the previous figure.

Figure 16: 

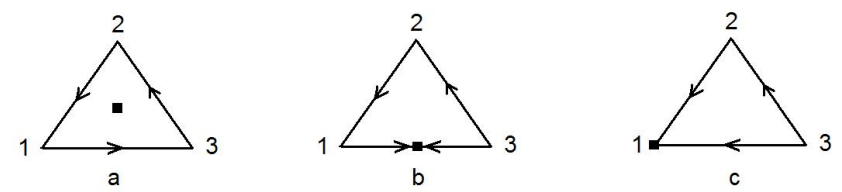

Figure 17: Effect of advection on competition in the Permanent Cyclic case II. As advection increases, the second species is the first to disappear.

Figure 18:

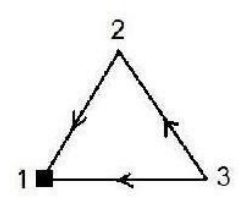

a

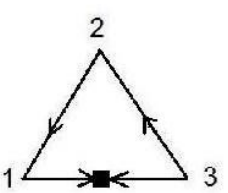

b

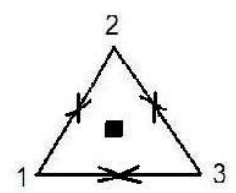

C

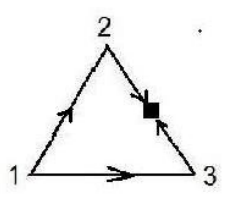

d

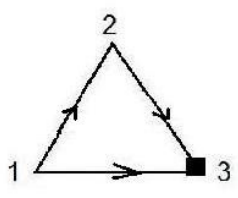

e

Figure 19: Effect of advection on competition in the Transitive case (a). The stable coexistence state of all three species is depicted in the middle triangle. Of course, not both of the subsystems involving species 2 need to have stable coexistence at the same time. The stable interior coexistence state exists for some other combinations as well (not shown).

Figure 20:

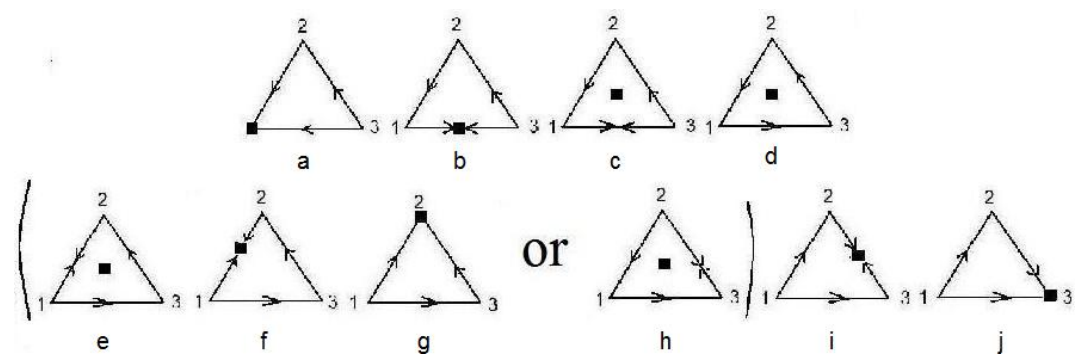

Figure 21: Effect of advection on competition in the Transitive case (b). Stages in brackets may or may not occur for different choices of parameters.

Figure 22: 


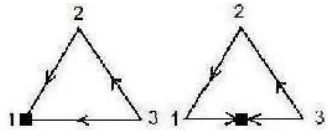

b

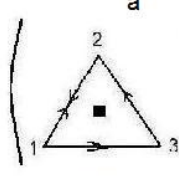

g

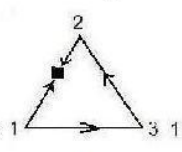

$\mathrm{h}$

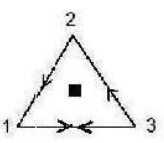

c

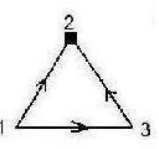

i

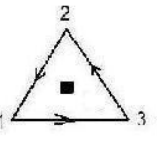

d

Or

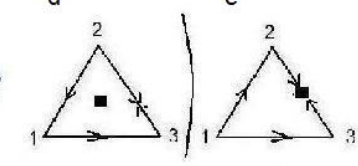

$\mathrm{k}$
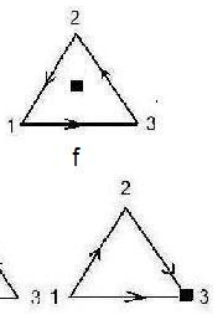

Figure 23: Effect of advection on competition in the Transitive case (c). The major difference to the previous plot is that the heterogeneous cycle is now stable for intermediate values of $\lambda$, see the fifth triangle in the top row.

Figure 24:
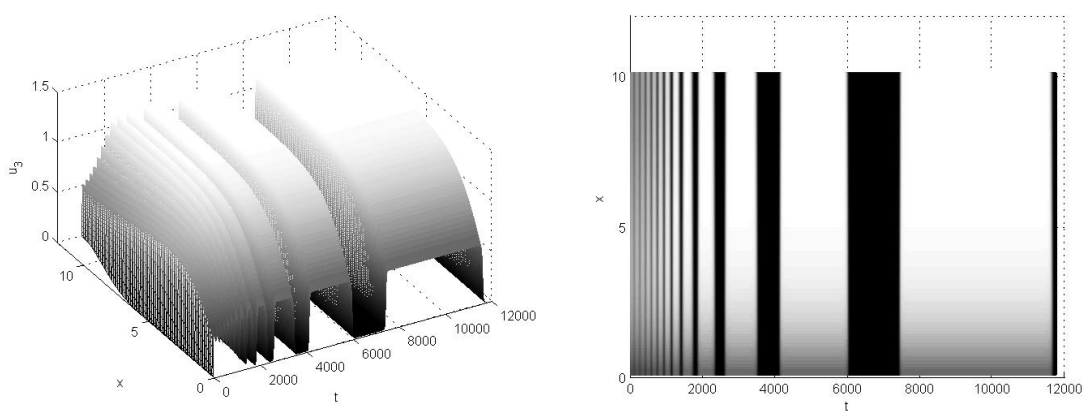

Figure 25:

Figure 26: The spatially explicit model shows an attractive heteroclinic cycle in the transitive case with $\alpha \beta>1$. Left: density of species 1 as a function of space and time. Right: Contour plot of the density of species 1 , where dark (light) represents low (high) density. In contrast to the previous figure, we can clearly see how the intervals of high and low density alternate periodically. Parameter values are $q=0.8, r_{1}=1, r_{2}=1.1, r_{3}=1.4, \alpha=0.69, \beta=1.5, L=10, d=1$. 